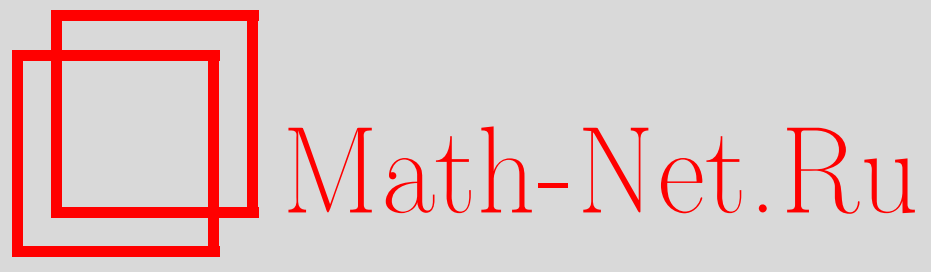

А. А. Доси, Спектр Тейлора и трансверсальность для операторной алгебры Гейзенберга, Матем. сб., 2010, том 201, номер 3, 39-62

DOI: https://doi.org/10.4213/sm7306

Использование Общероссийского математического портала Math-Net.Ru подразумевает, что вы прочитали и согласны с пользовательским соглашением http://www . mathnet.ru/rus/agreement

Параметры загрузки:

IP : 54.209 .52 .79

26 апреля 2023 г., 13:28:11

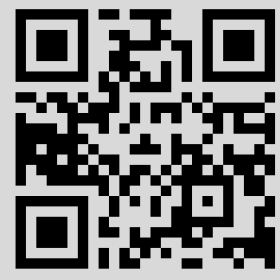




\title{
А. А. Доси \\ Спектр Тейлора и трансверсальность для операторной алгебры Гейзенберга
}

\begin{abstract}
Рассмотрены проблемы некоммутативного голоморфного функционального исчисления для банаховых модулей над конечномерными нильпотентными алгебрами Ли. Основной результат устанавливает свойство трансверсальности алгебр некоммутативных голоморфных функций по отношению к спектру Тейлора семейства ограниченных линейных операторов, порождающего алгебру Гейзенберга.
\end{abstract}

Библиография: 25 названий.

Ключевые слова: голоморфная функция от элементов алгебры Ли, спектр Тейлора, свойство трансверсальности, обращающее пополнение Фреше.

\section{§1. Введение}

Одним из оснований современного некоммутативного анализа и геометрии является понятие функции нескольких некоммутирующих переменных или ее обобщение - некоммутативная функциональная алгебра. Эти понятия мотивированы квантовой механикой, в которой в сравнении с классической механикой коммутирующие переменные заменены некоммутирующими операторами наблюдаемых.

Вопрос о реализации функциональной алгебры в виде алгебры операторов известен как проблема построения некоммутативного функционального исчисления. Под реализацией в виде алгебры операторов мы понимаем непрерывный гомоморфизм, вообще говоря, из некоммутативной функциональной алгебры $A$ в банахову алгебру $\mathscr{L}(X)$ всех ограниченных линейных операторов на некотором банаховом пространстве $X$. K примеру, в случае когда $T=\left(T_{1}, \ldots, T_{n}\right)$ семейство взаимно коммутирующих операторов в $\mathscr{L}(X)$ и $D \subseteq \mathbb{C}^{n}-$ некоторая область, непрерывный гомоморфизм $\mathscr{O}(D) \rightarrow \mathscr{L}(X)$ из алгебры Фреше $\mathscr{O}(D)$ всех голоморфных функций на $D$ в алгебру $\mathscr{L}(X)$, сопоставляющий координатной функции $z_{i}$ оператор $T_{i}$, называется голоморфным функииональным исчислением на $D$ для набора операторов $T$.

Используя методы топологической гомологии, Дж. Тейлор (см. [1]) рассмотрел проблему построения функционального исчисления в общем случае. Им был найден общий подход к этой проблеме, основные этапы которого выглядят следующим образом:

1) построение базовой (некоммутативной) алгебры полиномов $\mathscr{B}$;

2) рассмотрение банахова левого $\mathscr{B}$-модуля $X$; 
3) построение семейства топологических алгебр $\mathscr{A}(U)$ (некоммутативных) функций одновременно с каноническими гомоморфизмами $\iota_{U}: \mathscr{B} \rightarrow \mathscr{A}(U)$.

Проблема сводится к тому, чтобы выбрать алгебру $\mathscr{A}(U)$ таким образом, чтобы банахово пространство $X$ стало левым $\mathscr{A}(U)$-модулем, чья новая структура $\mathscr{B}$-модуля посредством гомоморфизма $\iota_{U}$ сводилась бы к исходной. Заметим, что для набора коммутирующих операторов $T=\left(T_{1}, \ldots, T_{n}\right)$ на банаховом пространстве $X$ базовая алгебра $\mathscr{B}$ есть алгебра $\mathscr{P}_{n}$ всех комплексных полиномов $n$ комплексных переменных $z=\left(z_{1}, \ldots, z_{n}\right)$ с канонической структурой

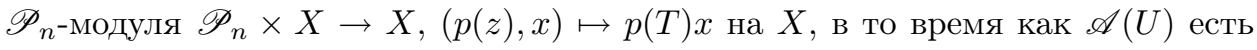
алгебра $\mathscr{O}(U)$ всех голоморфных функций в области $U \subseteq \mathbb{C}^{n}$. В общем случае семейство топологических алгебр $\mathscr{A}(U)$ обычно задается с помощью некоторого пучка (предпучка) Фреше $\mathscr{A}$. Сечения этого пучка над открытыми множествами $U$ и являются требуемыми алгебрами $\mathscr{A}(U)$.

Главной идеей этого подхода является установление тонкой связи между топологическими гомологиями и геометрическими характеристиками спектра. Кратко говоря, трансверсальное поведение $\mathscr{A}(U) \perp X$ алгебр $\mathscr{A}(U)$ относительно банахова модуля $X$ порождает резольвентные точки $\mathscr{B}$-модуля $X$. Отметим, что соотношение трансверсальности $\mathscr{A}(U) \perp X$ имеет чисто гомологическую природу, что выражается в равенстве нулю всех групп гомологий

$$
\operatorname{Tor}_{k}^{\mathscr{B}}(\mathscr{A}(U), X)=\{0\}, \quad k \geqslant 0 .
$$

В настоящей работе мы используем гомологическую теорию топологических алгебр, построенную Дж. Тейлором (см. [2]) и А. Я. Хелемским (см. [3]). Если в рамках этих теорий рассматривать пучок $\mathscr{O}$ ростков обычных голоморфных функций в $\mathbb{C}^{n}$, то дополнение резольвентных точек оказывается совместным спектром Тейлора $\sigma(T)$ (см. [1]) семейства коммутирующих операторов $T$. Отметим, что совместный спектр в настоящей работе относится к наборам ограниченных линейных операторов, действующих в банаховом пространстве, в то время как совместному спектру наборов дифференциальных операторов посвящен ряд работ В. П. Маслова и других авторов (см., например, монографию [4]). Напомним, что $\sigma(T)$ - это компактное подмножество в $\mathbb{C}^{n}$, состоящее из таких точек $a \in \mathbb{C}^{n}$, для которых комплекс Кошуля $\operatorname{Kos}(T-a, X)$, ассоциированный с набором $T-a$, не является точным (см. [5]). Центральный результат, полученный в [1] (см. также [6], [7]), утверждает, что если область $U$ содержит спектр Тейлора $\sigma(T)$, то набор операторов $T$ имеет голоморфное функциональное исчисление на $U$.

В случае некоммутативной базовой алгебры $\mathscr{B}$ сначала нужно построить спектральную теорию банаховых $\mathscr{B}$-модулей, а затем указать предпучок Фреше $\mathscr{A}$, который бы представил некоммутативные функции от элементов алгебры $\mathscr{B}$. Недавние достижения (см. [8]-[12]) спектральной теории нильпотентных операторных алгебр Ли открывают возможности применения метода Тейлора к универсальной обертывающей алгебре $\mathscr{B}=\mathscr{U}(\mathfrak{g})$ конечномерной нильпотентной алгебры Ли $\mathfrak{g}$. В частности, можно дать определение спектра Тейлора $\sigma(\mathfrak{g}, X)$ банахова $\mathscr{U}(\mathfrak{g})$-модуля $X$, обладающего свойством спектрального отображения по отношению к некоммутативным полиномам. 
Алгебры голоморфных функций от элементов нильпотентной алгебры Ли $\mathfrak{g}$ строятся как пополнения Аренса-Майкла универсальной обертывающей алгебры $\mathscr{U}(\mathfrak{g})$ (см. [13]-[15]). Напомним (см. [16]), что алгебра Аренса-Майкла определяется как полная топологическая алгебра, топология которой определяется мультипликативной системой полунорм.

Если $D_{a} \subseteq \mathbb{C}^{n}(n=\operatorname{dim}(\mathfrak{g}))$ - полидиск с центром в точке $a$, то алгебpa $\mathscr{O}_{\mathfrak{g}}\left(D_{a}\right)$ голоморфных функций в $D_{a}$ от элементов конечномерной алгебры Ли $\mathfrak{g}$ вводится как некоторое пополнение Фреше-Аренса-Майкла универсальной обертывающей алгебры $\mathscr{U}(\mathfrak{g})$. В результате $\mathscr{O}_{\mathfrak{g}}\left(D_{a}\right)$ разлагается в топологическую прямую сумму своего радикала Джекобсона $\operatorname{Rad} \mathscr{O}_{\mathfrak{g}}\left(D_{a}\right)$ и замкнутого подпространства $\mathscr{S}_{e}\left(D_{a}\right)$, которое топологически изоморфно алгебре $\mathscr{O}\left(D_{a} \cap \mathbb{C}^{m}\right), m=\operatorname{dim}(\mathfrak{g} /[\mathfrak{g}, \mathfrak{g}]):$

$$
\mathscr{O}_{\mathfrak{g}}\left(D_{a}\right)=\operatorname{Rad} \mathscr{O}_{\mathfrak{g}}\left(D_{a}\right) \oplus \mathscr{S}_{e}\left(D_{a}\right)
$$

В частности, мы получаем предпучок $\mathscr{O}_{\mathfrak{g}}$ некоммутативных алгебр Фреше над пространством $\mathbb{C}^{m}$ такой, что факторпредпучок $\mathscr{O}_{\mathfrak{g}} / \operatorname{Rad} \mathscr{O}_{\mathfrak{g}}$ сводится к пучку $\mathscr{O}$. Вопрос о том, является ли $\mathscr{O}_{\mathfrak{g}}$ пучком, остается открытым (см. [17], [18]). Ответ на него, по-видимому, зависит от структуры радикала $\operatorname{Rad} \mathscr{O}_{\mathfrak{g}}$.

Для того чтобы обойти эту трудность, можно пополнить предпучок $\mathscr{O}_{\mathfrak{g}}$. А именно, формальное пополнение Фреше $\mathfrak{T}_{\mathfrak{g}}$ предпучка $\mathscr{O}_{\mathfrak{g}}$ является пучком алгебры Фреше, который называется пучком ростков формальных радикальных функций от элементов алгебры Ли $\mathfrak{g}$ (см. [18], [19]). Как пучок пространств Фреше пучок $\mathfrak{T}_{\mathfrak{g}}$ имеет относительно простую структуру. А именно, он совпадает с проективным тензорным произведением $\mathfrak{T}_{\mathfrak{g}}=\mathscr{O} \widehat{\otimes} \mathbb{C}\left[\left[\omega_{1}, \ldots, \omega_{n-m}\right]\right]$ пучка $\mathscr{O}$ и постоянного пучка $\mathbb{C}\left[\left[\omega_{1}, \ldots, \omega_{n-m}\right]\right]$ алгебры формальных степенных рядов от $n-m$ комплексных переменных. Таким образом, алгебра $\operatorname{Rad} \mathfrak{T}_{\mathfrak{g}}$ является алгеброй формальных степенных рядов. Отметим, что близкая к этой конструкция используется в некоммутативной алгебраической геометрии (см. [20]).

$\mathrm{K}$ сожалению, формальное пополнение предпучка $\mathscr{O}_{\mathfrak{g}}$ существенно ограничивает соответствующий класс банаховых $\mathfrak{g}$-модулей (или наборов операторов), которые можно рассматривать для решения проблемы построения функционального исчисления. Точнее, приходится иметь дело со строгими банаховыми $\mathfrak{g}$-модулями $X$ в том смысле, что коммутаторы из $[\mathfrak{g}, \mathfrak{g}]$ действуют на $X$ как нильпотентные операторы (в общем случае они квазинильпотентны). В [21] и [22] функциональное исчисление Тейлора было расширено следующим образом: если $X$ является строгим банаховым $\mathfrak{g}$-модулем и $U-$ область в $\mathbb{C}^{m}$, содержащая спектр Тейлора $\sigma(\mathfrak{g}, X)$, то существует непрерывный гомоморфизм алгебр $\mathfrak{T}_{\mathfrak{g}}(U) \rightarrow X$, переносящий структуру банахова $\mathscr{U}(\mathfrak{g})$-модуля на $X$.

В настоящей работе исследуется проблема построения функционального исчисления для банахова $\mathfrak{g}$-модуля $X$. Центральный этап исследований заключается в изучении свойства трансверсальности алгебр Фреше-Аренса-Майкла $\mathscr{O}_{\mathfrak{g}}\left(D_{a}\right)$ по отношению к банахову $\mathfrak{g}$-модулю $X$. Иными словами, изучаются достаточные условия для того, чтобы соотношение $\mathscr{O}_{\mathfrak{g}}\left(D_{a}\right) \perp X$ влекло включение точки $a$ в резольвентное множество $\mathbb{C}^{m} \backslash \sigma(\mathfrak{g}, X)$. В [23] и [24] свойство трансверсальности рассматривалось для алгебры $\mathscr{O}_{\mathfrak{g}}\left(\mathbb{C}^{m}\right)$ всех целых функций от 
элементов нильпотентной алгебры Ли $\mathfrak{g}$, рост которой нормален (см. [17]), т.е. степени коммутаторов убывают с некоторой (нормальной) скоростью. Аналогично коммутативному случаю нормальный рост нильпотентной алгебры Ли $\mathfrak{g}$ позволяет доказать существование абсолютного базиса в $\mathscr{O}_{\mathfrak{g}}\left(\mathbb{C}^{n}\right)($ см. [17]).

Эта техника неприменима для алгебр $\mathscr{O}_{\mathfrak{g}}\left(D_{a}\right)$ функций в полидиске $D_{a}$, отличном от $\mathbb{C}^{n}$. В этом случае существует другой базис в $\mathscr{O}_{\mathfrak{g}}\left(D_{a}\right)$ (см. [14]), о котором не известно, является он безусловным или нет. Это существенно отличает ситуацию от случая коммутативных алгебр. Тем не менее мы доказываем, что свойство трансверсальности имеет место для алгебры Гейзенберга, которая является ключевым примером алгебры Ли с нормальным ростом.

Напомним, что алгебра Гейзенберга $\mathfrak{g}$ - это двухступенчатая алгебра Ли с каноническим базисом $e_{1}, e_{2}, e_{3}$ и соотношениями

$$
\left[e_{1}, e_{2}\right]=e_{3}, \quad\left[e_{1}, e_{3}\right]=\left[e_{2}, e_{3}\right]=0
$$

Если $X$ - банахов $\mathfrak{g}$-модуль, то в нем имеются ограниченные операторы $T$ и $S$, реализующие действия генераторов $e_{1}$ и $e_{2}$. Иными словами,

$$
[T,[T, S]]=[S,[T, S]]=0 .
$$

Любая пара коммутирующих операторов $T$ и $S$ (иначе говоря, таких, что $[T, S]=0)$ порождает алгебру Гейзенберга. Отметим, что в случае, когда пара $(T, S)$ порождает некоммутативную алгебру Гейзенберга, ее коммутатор $[T, S]$ является нетривиальным квазинильпотентным оператором в $\mathscr{L}(X)$. Этот факт является простым следствием известной формулы Кляйнеке-Широкова (см. ниже формулу (1)). Если $[T, S]$ оказывается нильпотентным оператором, то пространство $X$ оказывается строгим банаховым $\mathfrak{g}$-модулем, что полностью решает проблему, как отмечалось выше.

Мы предлагаем операторный подход (см. также [24] и [18]) для решения вопроса о трансверсальности. Его суть состоит в обращении оператора правого умножения на $\mathscr{U}(\mathfrak{g})$ над топологическим пополнением пространства $\mathscr{U}(\mathfrak{g})$. Главный результат работы утверждает, что операторный набор правого умножения на $\mathscr{O}_{\mathfrak{g}}\left(D_{a}\right)$ локально обратим слева. Более точно, пусть $X$ - пространство $\Phi$ реше, $\mathscr{L}(X)$ - пространство всех непрерывных линейных операторов на $X$ и $T=\left(T_{1}, \ldots, T_{n}\right)$ - набор операторов в $\mathscr{L}(X)$. Набор $T$ называется локально обратимым слева на $X$, если существуют непрерывные проекторы

$$
1=P_{0} \geqslant P_{1} \geqslant \cdots \geqslant P_{n}
$$

такие, что

$$
T_{k} P_{k-1}=P_{k-1} T_{k} P_{k-1}
$$

и $T_{k} \mid \operatorname{im}\left(P_{k-1}\right)$ является биекцией на $\operatorname{im}\left(P_{k-1}\right) \cap \operatorname{im}\left(1-P_{k}\right)$ для всех значений индекса $k$ (см. определение в п. 3.1). Пусть $Y$ есть локально выпуклое пространство, в котором выделено непрерывное линейное отображение $\iota: Y \rightarrow X$ с плотным образом в $X$. Операторный набор $t=\left(t_{1}, \ldots, t_{n}\right)$ в $\mathscr{L}(Y)$ называется 
ı-совместимым, если диаграмма

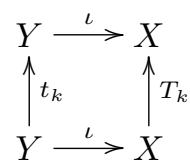

коммутативна для всех значений $k$.

Мы говорим, что отображение $\iota$ является $t$-обращающим, если набор $T$ локально обратим слева на $X$. Таким образом, если набор $t$ локально обратим на $Y$ и его канонический обратный является $\iota$-совместимым, то набор $T$ локально обратим на $X$ (см. теорему 1$)$. Далее мы применяем этот результат к случаю $Y=\mathscr{U}(\mathfrak{g})$, где топология в $Y$ - сильнейшая локально выпуклая топология, и к случаю алгебры Фреше $X=\mathscr{O}_{\mathfrak{g}}\left(D_{a}\right)$, для которой $\iota: \mathscr{U}(\mathfrak{g}) \rightarrow \mathscr{O}_{\mathfrak{g}}\left(D_{a}\right)$ есть каноническое вложение с плотным образом. Отметим, что все операторы правого умножения $R_{u} \in \mathscr{L}(\mathscr{U}(\mathfrak{g})), u \in \mathfrak{g}$, являются $\iota$-совместимыми. Мы показываем, что если $\mathfrak{g}$ есть алгебра Гейзенберга с каноническим базисом $e=\left(e_{1}, e_{2}, e_{3}\right)$, то набор $T_{e}=\left(R_{e_{3}}, R_{e_{2}}, R_{e_{1}}\right)$ локально обратим слева на $\mathscr{O}_{\mathfrak{g}}\left(D_{a}\right)$. Отсюда следует главный результат статьи о трансверсальности.

Пользуюсь случаем выразить свою благодарность Ю.В. Туровскому и А. Ю. Пирковскому за полезные обсуждения.

\section{§ 2. Предварительные сведения}

Мы предполагаем, что все рассмотренные линейные пространства являются комплексными, а ассоциативные алгебры - унитальными. Если $X$ - конечномерное линейное пространство с базисом $e=\left(e_{1}, \ldots, e_{n}\right)$, то $\ell_{1}$-норму на $X$ по отношению к базису е обозначим через $l_{e}: l_{e}\left(\sum a_{i} e_{i}\right)=\sum\left|a_{i}\right|, a_{i} \in \mathbb{C}$. Пусть $\mathbb{R}_{+}^{n}-n$-я декартова степень множества $\mathbb{R}_{+}$всех положительных действительных чисел, и пусть $\overline{\mathbb{R}}_{+}=\mathbb{R}_{+} \cup\{+\infty\}$. Для $s, t \in \mathbb{R}_{+}^{n}$ положим $s \leqslant t$, если $s_{i} \leqslant t_{i}$ для всех $i, 1 \leqslant i \leqslant n$, где $s_{i}=s(i), t_{i}=t(i)$. Очевидно, что $\mathbb{R}_{+}^{n}$, снабженное отношением $\leqslant$, частично упорядочено. Говорят, что направленное подмножество $S \subseteq \mathbb{R}_{+}^{n}$ является неограниченно возрастающим множеством, если для каждого положительного целого $k \in \mathbb{N}$ существует $s^{(k)} \in S$ такое, что $\min \left(s^{(k)}\right) \geqslant k$. Если $s \in \mathbb{R}_{+}^{n}$, то $\ell_{1}$-норму на $X$ относительно базиса $s^{-1} e=\left(s_{1}^{-1} e_{1}, \ldots, s_{n}^{-1} e_{n}\right)$ обозначим через $l_{e, s}$.

В случае, когда $X$ - линейное пространство, $\wedge X=\bigoplus_{k \geqslant 0} \wedge^{k} X$ обозначает внешнюю алгебру пространства $X$. Если $\underline{u}=u_{1} \wedge \cdots \wedge u_{k} \in \wedge^{k} X-k$-вектор, то мы будем использовать обозначение $\underline{u}_{i}=u_{1} \wedge \cdots \wedge \widehat{u}_{i} \wedge \cdots \wedge u_{k}$ для $(k-1)$ вектора, в выражении которого $\widehat{u}_{i}$ обозначает пропуск переменной $u_{i}$. Если отбрасываются две переменные, $u_{i}$ и $u_{j}, i<j$, в выражения $\underline{u}$, то полученный вектор обозначим через $\underline{u}_{i j}$.

Мы используем обычное обозначение $X \widehat{\otimes} Y$ для проективного тензорного произведения (хаусдорфовых) полинормированных (или локально выпуклых) пространств $X$ и $Y$. Говорят, что непрерывное линейное отображение 
$T: X \rightarrow Y$ является ретракцией (коретракцией), если $T S=1$ (соответственно $S T=1)$ для некоторого непрерывного линейного отображения $S: Y \rightarrow X$. В этом случае $Y$ называется ретрактом (коретрактом) $X$.

Радикал Джекобсона ассоциативной алгебры $A$ обозначается через $\operatorname{Rad} A$. Левый (соответственно правый) оператор умножения в $A$ обозначается через $L_{a}$ (соответственно через $R_{a}$ ). По определению $L_{a}(x)=a x$ и $R_{a}(x)=x a$ для всех $a, x \in A$. Единица в алгебре $A$ обозначается через $1_{A}$, а противоположная алгебра к $A$ - через $A^{\text {оp }}$. Скажем, что $A$ - топологическая алгебра, если она является полинормированным пространством с раздельно непрерывным мультипликативным отображением $A \times A \rightarrow A$. Говорят, что алгебра $A$ является $\widehat{\otimes}$-алгеброй (см. [2]), если она представляет собой полную полинормированную топологическую алгебру с совместно непрерывным умножением $A \times A \rightarrow A$. Если топология $\widehat{\otimes}$-алгебры порождается семейством мультипликативных полунорм, то $A$ называется алгеброй Аренса-Майкла (см. [16; п. 1.2.4]). Алгебра $A$ с выделенным непрерывным характером (мультипликативным линейным функционалом) $\varepsilon_{A}: A \rightarrow \mathbb{C}$ называется отмеченной алгеброй. Далее, говорят, что полинормированное пространство $X$ является левым полинормированным модулем или левым $\widehat{\otimes}$-модулем над $A$, если $X$ имеет структуру левого $A$-модуля такую, что отображение $A \times X \rightarrow X,(a, x) \mapsto a \cdot x$, является совместно непрерывным. Аналогично определяется правый модуль (бимодуль) над $A$. Категория (к которой мы обычно будем относиться как к классу) всех левых $\widehat{\otimes}$-модулей над $A$ обозначается через $A$-mod. Точно так же $\bmod -A$ (coответственно $A$-mod- $A$ ) обозначает категорию всех правых модулей (соответственно бимодулей). Говорят, что цепной комплекс

$$
(\mathscr{X}, d): \cdots \longleftarrow X_{n-1} \stackrel{d_{n-1}}{\longleftarrow} X_{n} \stackrel{d_{n}}{\longleftarrow} X_{n+1} \longleftarrow \cdots
$$

в категории $A-\bmod ($ или $\bmod -A$ ) является допустимым, если он расщепляется как комплекс полинормированных пространств. Если $A$ - отмеченная алгебра с выделенным непрерывным характером $\varepsilon_{A}: A \rightarrow \mathbb{C}$, то одномерное пространство $\mathbb{C}$ превращается в $A$-модуль вдоль $\varepsilon_{A}$; этот $A$-модуль называется $m p и в и$ альным и обозначается через $\mathbb{C}\left(\varepsilon_{A}\right)$.

Универсальная обертывающая алгебра конечномерной алгебры Ли $\mathfrak{g}$ обозначается как $\mathscr{U}(\mathfrak{g})$. Алгебра $\mathscr{U}(\mathfrak{g})$ превращается в топологическую, если снабдить ее сильнейшей полинормированной топологией.

Известно (см. [25; п. 2.2.7]), что отображение

$$
\varkappa_{\mathfrak{g}}: \mathscr{U}(\mathfrak{g}) \rightarrow \mathscr{U}(\mathfrak{g})^{\mathrm{op}}, \quad \varkappa_{\mathfrak{g}}(x)=-x, \quad x \in \mathfrak{g},
$$

является алгебраическим изоморфизмом, который называется антиподом алгебры $\mathscr{U}(\mathfrak{g})$, и $\mathscr{U}(\mathfrak{g})^{\text {op }}=\mathscr{U}\left(\mathfrak{g}^{\text {op }}\right)$. Пространство всех характеров алгебры Ли $\mathfrak{g}$ обозначается через $\Delta(\mathfrak{g})$. Пространство всех характеров алгебры $\mathscr{U}(\mathfrak{g})$ отождествляется с $\Delta(\mathfrak{g})$, т.е. каждый характер алгебры Ли $\lambda \in \Delta(\mathfrak{g})$ имеет единственное расширение до характера алгебры $\mathscr{U}(\mathfrak{g})$, которое также обозначается через $\lambda$. В частности, алгебра $\mathscr{U}(\mathfrak{g})$ имеет характер $\varepsilon_{\mathfrak{g}}: \mathscr{U}(\mathfrak{g}) \rightarrow \mathbb{C}$, который расширяет тривиальный гомоморфизм Ли $\mathfrak{g} \rightarrow \mathbb{C}$. Таким образом, 
$\mathscr{U}(\mathfrak{g})$ является отмеченной алгеброй с тривиальным характером $\varepsilon_{\mathfrak{g}}$. Пусть $\mathfrak{g}$ является конечномерной нильпотентной алгеброй Ли с обрывающимся нижним центральным рядом $\left\{\mathfrak{g}^{(s)}: s \geqslant 1\right\}, \mathfrak{g}^{(1)}=\mathfrak{g}, \mathfrak{g}^{(s)}=\left[\mathfrak{g}, \mathfrak{g}^{(s-1)}\right], s>1$. Говорят, что базис $e=\left(e_{1}, \ldots, e_{n}\right)$ в $\mathfrak{g}$ является треугольным базисом, если он подчинен нижнему центральному ряду. Таковым является канонический базис $e=\left(e_{1}, e_{2}, e_{3}\right)$ алгебры Гейзенберга $\mathfrak{g}$, удовлетворяющей тождествам $\left[e_{1}, e_{2}\right]=e_{3},\left[e_{1}, e_{3}\right]=\left[e_{2}, e_{3}\right]=0$.

Возьмем базис $e=\left(e_{1}, \ldots, e_{n}\right)$ алгебры Ли g. Для $n$-набора $J=\left(j_{1}, \ldots, j_{n}\right) \in$ $\mathbb{Z}_{+}^{n}$ неотрицательных целых чисел мы положим $e^{J}=e_{1}^{j_{1}} \cdots e_{n}^{j_{n}}$ для указания упорядоченного монома в $\mathscr{U}(\mathfrak{g})$, взятого по базису $e$. По теореме ПуанкареБиркгофа-Витта множество $\left\{e^{J}\right\} \subseteq \mathscr{U}(\mathfrak{g})$ всех упорядоченных мономов является алгебраическим базисом (см. [25; п. 2.2.1]) в $\mathscr{U}(\mathfrak{g})$. Отсюда мы получаем корректно определенные (относительно базиса $е$ ) операторы деления

$$
\nabla_{e_{i}} \in \mathscr{L}(\mathscr{U}(\mathfrak{g})), \quad \nabla_{e_{i}}\left(e_{1}^{j_{1}} \cdots e_{n}^{j_{n}}\right)=\delta_{j_{i}} e_{1}^{j_{1}} \cdots e_{i}^{j_{i}-1} \cdots e_{n}^{j_{n}},
$$

где

$$
\delta_{j_{i}}= \begin{cases}1, & \text { если } j_{i+1}+\cdots+j_{n}<1 \leqslant j_{i}, \\ 0 & \text { в противном случае. }\end{cases}
$$

Наконец, пусть $\mathfrak{g}$ - алгебра Гейзенберга с ее каноническим базисом $e=$ $\left(e_{1}, e_{2}, e_{3}\right)$, и предположим, что $\mathfrak{g}$ является подалгеброй Ли некоторой ассоциативной банаховой алгебры $A$. Тогда справедлива формула $k ! e_{3}^{k}=\operatorname{ad}\left(e_{1}\right)^{k}\left(e_{2}^{k}\right)$, $k \in \mathbb{N}$, где $\operatorname{ad}\left(e_{1}\right) \in \mathscr{L}(A), \operatorname{ad}\left(e_{1}\right)=L_{e_{1}}-R_{e_{1}}$, которая может быть легко проверена с помощью индукции по $k$. Из этого следует, что

$$
\left\|e_{3}^{k}\right\|_{A} \leqslant(k !)^{-1}\left\|\operatorname{ad}\left(e_{1}\right)\right\|_{A}^{k}\left\|e_{2}\right\|_{A}^{k} \leqslant(k !)^{-1} 2^{k}\left\|e_{1}\right\|_{A}^{k}\left\|e_{2}\right\|_{A}^{k}
$$

или $\left\|e_{3}^{k}\right\|_{A}^{1 / k} \leqslant(k !)^{-1 / k} \gamma_{A}$, где $\gamma_{A}=2\left\|e_{1}\right\|_{A}\left\|e_{2}\right\|_{A}$. Но $(k !)^{-1 / k} \sim k^{-1}$ с точностью до известной константы в силу формулы Стирлинга. Получаем, что $e_{3}$ является квазинильпотентным элементом алгебры $A$ и

$$
\left\|e_{3}^{k}\right\|_{A}^{1 / k}=O\left(k^{-1}\right) .
$$

Похожее поведение степеней элементов из $\mathfrak{g}^{(2)}$ наблюдается во многих других нильпотентных алгебрах Ли. Такие алгебры Ли характеризуются в [17] как класс нильпотентных алгебр Ли с нормальным ростом. Таким образом, каждая алгебра Гейзенберга является нильпотентной алгеброй Ли с нормальным ростом.

\section{§ 3. Обращающее пополнение Фреше}

В этом параграфе мы кратко обрисуем метод обращающих пополнений Фреше полинормированного пространства, предложенный в [24], который определяет операторный подход к некоммутативной задаче локализации в рамках класса алгебр Фреше. 
3.1. Локально обратимые слева операторные наборы. Пусть $X$ пространство Фреше. Под проектором в $X$ мы понимаем непрерывный опеpaтор $P \in \mathscr{L}(X)$ такой, что $P^{2}=P$. Если $P$ является проектором в $X$, то $1-P$ - также проектор, обозначаемый как $P^{c}$. Если $P$ и $Q$ являются проекторами в $X$, то пишем $P \leqslant Q$, если $P=Q P$ и $\operatorname{im}\left(P^{c} Q\right) \subseteq \operatorname{im}(Q)$. Заметим, что $P \leqslant Q$ тогда и только тогда, когда

$$
\operatorname{im}(P) \subseteq \operatorname{im}(Q), \quad \operatorname{im}(Q)=\left(\operatorname{im}(Q) \cap \operatorname{im}\left(P^{c}\right)\right) \oplus \operatorname{im}(P)
$$

(см. [24]).

ОПРеДЕЛЕНИЕ. Пусть $X$ является пространством Фреше, и пусть $T=\left(T_{1}\right.$, $\left.\ldots, T_{n}\right)$ - набор из $n$ элементов пространства $\mathscr{L}(X)$. Мы говорим, что $T$ локально обратим слева в $X$, если существуют проекторы $1=P_{0} \geqslant P_{1} \geqslant \cdots \geqslant P_{n}$ в $\mathscr{L}(X)$ такие, что $T_{k} P_{k-1}=P_{k-1} T_{k} P_{k-1}$ и $T_{k} \mid \operatorname{im}\left(P_{k-1}\right)$ является биекцией на $\operatorname{im}\left(P_{k-1}\right) \cap \operatorname{im}\left(P_{k}^{c}\right)$ для любого $k$.

Пусть $S_{k}$ обратный к оператору $T_{k}: \operatorname{im}\left(P_{k-1}\right) \rightarrow \operatorname{im}\left(P_{k-1}\right) \cap \operatorname{im}\left(P_{k}^{c}\right)$. Pacширяя его очевидным способом на $\operatorname{im}\left(P_{k}\right)$ (или на все пространство $X$ ), мы получим левый обратный $S_{k}: \operatorname{im}\left(P_{k-1}\right) \rightarrow \operatorname{im}\left(P_{k-1}\right)$ оператора $T_{k}: \operatorname{im}\left(P_{k-1}\right) \rightarrow$ $\operatorname{im}\left(P_{k-1}\right)$ для любого $k$. Скажем тогда, что набор $S=\left(S_{1}, \ldots, S_{n}\right)$ есть канонический (левыци) обратный набора $T$ по отношению проекторов $P_{1}, \ldots, P_{n}$.

Заметим, что локально обратимые слева операторные наборы могут быть рассмотрены также в чисто алгебраическом смысле, поскольку то же определение имеет смысл для линейного пространства $X$ (снабженного сильнейшей полинормированной топологией) и набора $n$ линейных операторов $T=$ $\left(T_{1}, \ldots, T_{n}\right)$ в пространстве $X$.

Таким образом, одноэлементный набор $T$, отождествляемый с оператором, является локально обратимым слева, если он имеет непрерывный левый обратный оператор $S$. Если $T=\left(T_{1}, T_{2}\right)$ - операторная пара в $\mathscr{L}(X)$, то $T$ является локально обратимой слева, если отображения в строках диаграммы

$$
\begin{aligned}
X \stackrel{T_{1}}{\rightarrow} \operatorname{im}\left(P_{1}^{c}\right) & \\
& \operatorname{im}\left(P_{1}\right) \stackrel{T_{2}}{\longrightarrow} \\
& \operatorname{im}\left(P_{1}\right) \cap \operatorname{im}\left(P_{2}^{c}\right) \\
& \oplus \\
& \operatorname{im}\left(P_{2}\right)
\end{aligned}
$$

представляют собой топологические изоморфизмы.

Наконец, отметим, что если набор $T=\left(T_{1}, \ldots, T_{n}\right)$ локально обратим слева в $X$, то локально обратим слева набор $T^{\prime}=\left(T_{1}, \ldots, T_{k}\right)$ для каждого $k, k \geqslant 1$, что легко следует из приведенного выше определения.

Пусть теперь $Y$ - полинормированное пространство, $X$ - пространство Фреше, и пусть $\iota: Y \rightarrow X-$ непрерывное линейное отображение, образ которого плотен. Мы скажем, что непрерывный линейный оператор $t: Y \rightarrow Y$ является 
$\iota$-совместимым, если $T \iota=\iota t$, т.е. если диаграмма

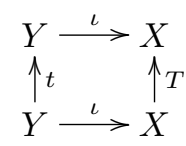

коммутативна для некоторого $T \in \mathscr{L}(X)$. В этом случае $T$ единствен, поскольку $\iota$ имеет плотный образ. Более того, множество $\mathscr{L}_{\iota}(Y)$ всех $\iota$-совместимых отображений является линейным подпространством в $\mathscr{L}(X)$ и соответствие $t \mapsto T$ является корректно определенным линейным отображением $\iota^{\prime}: \mathscr{L}_{\iota}(Y) \rightarrow$ $\mathscr{L}(X)$. Если $t=\left(t_{1}, \ldots, t_{n}\right)$ является набором $n$ элементов в $\mathscr{L}_{\iota}(Y)$, то мы положим $T=\left(T_{1}, \ldots, T_{n}\right)$ в качестве набора $n$ элементов $\iota^{\prime}(t)=\left(\iota^{\prime}\left(t_{1}\right), \ldots, \iota^{\prime}\left(t_{n}\right)\right)$ B $\mathscr{L}(X)$.

Пусть теперь $t=\left(t_{1}, \ldots, t_{n}\right)$ является набором $n$ элементов пространства $\mathscr{L}_{\iota}(Y)$. Мы скажем, что отображение $\iota$ является $t$-обращающим, если $T$ является локально обратимым слева в $X$. Задача обращения Фреше $n$-набора $t=\left(t_{1}, \ldots, t_{n}\right)$ в $\mathscr{L}(Y)$ заключается в нахождении $t$-обращающего отображения $\iota: Y \rightarrow X$ в некоторое пространство Фреше $X$.

В такой общей форме эта задача возникает во многих случаях. $\mathrm{K}$ примеру, зафиксируем любое линейное пространство $Y$, обладающее сильнейшей полинормированной топологией, и зафиксируем набор $t=\left(t_{1}, \ldots, t_{n}\right)$ в $\mathscr{L}(Y)$. Как получить пополнение Фреше $X$ от $Y$ такое, чтобы каноническое вложение $Y \rightarrow X$ было $t$-обращающим? Для одноэлементного набора $t \in \mathscr{L}(Y)$ задача может быть решена, к примеру, следующим образом. Предположим, что $X-$ пополнение Фреше пространства $Y$ такое, что $t$ непрерывно в топологии, наследуемой от $X$, и пусть $T \in \mathscr{L}(X)$ - его непрерывное расширение. Если $t$ имеет левый обратный, скажем, $s \in \mathscr{L}(Y)$, который непрерывен в топологии пространства $X$, то непрерывное расширение $S \in \mathscr{L}(X)$ оператора $s$ будет левым обратным для $T$, т.е. $S T=1$. Последнее в свою очередь означает, что $T$ является локально обратимым слева.

В общем случае нескольких "переменных" справедливо следующее утверждение, доказанное в [24].

ТеОремА 1. Пусть $Y$ - полинормированное пространство, $X$ - пространство Фреше, $\iota: Y \rightarrow X-$ - непрерывное линейное отображение, чей образ является плотным, и пусть $t$ является набором $n$ ь-совместимых операторов из $\mathscr{L}(Y)$. Если $t$ является локально обратимым слева в $Y$ и его канонический левый обратный является ь-совместимым, то $T$ локально обратим слева в $X$.

3.2. Обращающее пополнение Фреше алгебры $\mathscr{U}(\mathfrak{g})$. Будем считать теперь, что пространство $Y=\mathscr{U}(\mathfrak{g})$ снабжено сильнейшей полинормированной топологией. Под пополнением обертывающей алгебры $\mathscr{U}(\mathfrak{g})$ мы подразумеваем некоторую алгебру Фреше $A$ с гомоморфизмом алгебр $\iota: \mathscr{U}(\mathfrak{g}) \rightarrow A$, чей образ является плотным в $A$. Мы будем обращать в $A$ операторы правого умножения из $\mathscr{L}(\mathscr{U}(\mathfrak{g}))$. Все левые операторы умножения $L_{u} \in \mathscr{L}(\mathscr{U}(\mathfrak{g}))$ (соответственно $\left.R_{u} \in \mathscr{L}(\mathscr{U}(\mathfrak{g}))\right)$ являются $\iota$-совместимыми, если $\iota$ является 
гомоморфизмом. Поэтому $\left\{L_{u}, R_{u}: u \in \mathfrak{g}\right\} \subseteq \mathscr{L}_{\iota}(\mathscr{U}(\mathfrak{g}))$. Возьмем треугольный базис $e=\left(e_{1}, \ldots, e_{n}\right)$ в $\mathfrak{g}$ и рассмотрим набор $t_{e}=\left(t_{1}, \ldots, t_{n}\right)$ элементов из $\mathscr{L}_{\iota}(\mathscr{U}(\mathfrak{g}))$, при этом пусть $t_{i}=R_{e_{n-i+1}} \in \mathscr{L}_{\iota}(\mathscr{U}(\mathfrak{g})), 1 \leqslant i \leqslant n$, взяты от операторов правого умножения в обратном порядке. Положим

$$
T_{e}=\left(T_{1}, \ldots, T_{n}\right) \quad \text { в } \mathscr{L}(A), \quad \text { где } T_{i}=R_{e_{n-i+1}}, \quad 1 \leqslant i \leqslant n .
$$

Скажем, что $A$ является (слабо) обращающим пополнением алгебры $\mathscr{U}(\mathfrak{g})$ (см. [24]), если набор $T_{e}$ является локально обратимым слева в $A$ для некоторого треугольного базиса $e$ в $\mathfrak{g}$, т.е. отображение $\iota: \mathscr{U}(\mathfrak{g}) \rightarrow A$ является $t_{e}$-обращающим. Рассмотрим подпространство $\mathscr{U}_{i}^{e}=\left\{\sum_{J} a_{J} e_{1}^{j_{1}} \cdots e_{i}^{j_{i}}: a_{J} \in \mathbb{C}\right\}$ в $\mathscr{U}(\mathfrak{g})$, и пусть $\mathscr{J}_{i}^{e}$ является идеалом в $\mathscr{U}(\mathfrak{g})$, порожденным $e_{i+1}, \ldots, e_{n}$. Тогда $\mathscr{U}(\mathfrak{g})=\mathscr{U}_{i}^{e} \oplus \mathscr{J}_{i}{ }^{e}$, так что существует проектор $p_{n-i}$ на $\mathscr{U}_{i}^{e}$ вдоль $\mathscr{J}_{i}^{e}$ для каждого $i$.

ЛЕмма 1. Набор из $n$ элементов $t_{e}$ является локально обратимым слева в $\mathscr{U}(\mathfrak{g})$ относительно проекторов $p_{1}, \ldots, p_{n}$ и набор $\nabla_{e}=\left(\nabla_{e_{n}}, \ldots, \nabla_{e_{1}}\right)$ в $\mathscr{L}(\mathscr{U}(\mathfrak{g}))$, составленный из операторов деления, является каноническим левым обратным.

ДоказАтельство. Очевидно, что $\mathscr{U}_{1}^{e} \subseteq \mathscr{U}_{2}^{e} \subseteq \cdots \subseteq \mathscr{U}_{n}^{e}=\mathscr{U}(\mathfrak{g})$ и что оператор деления $\nabla_{e_{i}}$ оставляет инвариантным подпространство $\mathscr{U}_{i}{ }^{e}$ для каждого $i$. По определению $p_{k}$ является проектором на $\mathscr{U}_{n-k}^{e}$ вдоль $\mathscr{J}_{n-k}^{e}, 0 \leqslant k \leqslant n$. Так как

$$
\mathscr{U}_{n-k+1}^{e}=\mathscr{U}_{n-k}^{e} \oplus\left(\mathscr{U}_{n-k+1}^{e} \cap \mathscr{J}_{n-k}^{e}\right)
$$

и $\mathscr{U}_{n-k+1}^{e} \cap \mathscr{J}_{n-k}^{e}$ содержит все полиномы $\sum_{J} a_{J} e^{J} \in \mathscr{U}_{n-k+1}^{e}$ с $j_{n-k+1} \geqslant 1$, получим, что

$$
\operatorname{im}\left(p_{k-1}\right)=\operatorname{im}\left(p_{k}\right) \oplus\left(\operatorname{im}\left(p_{k-1}\right) \cap \operatorname{im}\left(p_{k}^{c}\right)\right) .
$$

Имеет место $p_{k-1} \geqslant p_{k}$ для всех $k$. Таким образом, $1=p_{0} \geqslant p_{1} \geqslant \cdots \geqslant p_{n}$. Более того, оператор $t_{k}$, будучи правым умножением на $e_{n-k+1}$, оставляет инвариантным $\mathscr{U}_{n-k+1}^{e}$, т.е. $t_{k} p_{k-1}=p_{k-1} t_{k} p_{k-1}$. Кроме того,

$$
t_{k}\left(\operatorname{im}\left(p_{k-1}\right)\right)=t_{k}\left(\mathscr{U}_{n-k+1}^{e}\right) \subseteq \mathscr{U}_{n-k+1}^{e} \cap \mathscr{J}_{n-k}^{e}=\operatorname{im}\left(p_{k-1}\right) \cap \operatorname{im}\left(p_{k}^{c}\right)
$$

и $\nabla_{e_{n-k+1}} t_{k}=\nabla_{e_{n-k+1}} R_{e_{n-k+1}}=1$ в $\mathscr{U}_{n-k+1}^{e}$ для всех $k$. По определению из п. $3.1 R_{e}$ является локально обратимым слева с его каноническим левым обратным $\nabla_{e}$.

Лемма 1 доказана.

СлеДСТвИЕ 1. Пусть $X$ - пополнение алгебры $\mathscr{U}(\mathfrak{g})$ с каноническим отоб-

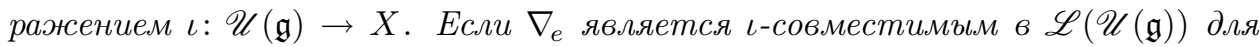
некоторого треугольного базиса е в $\mathfrak{g}$, то $X$ является обращаюоим пополнением пространства $\mathscr{U}(\mathfrak{g})$.

Для доказательства достаточно применить теорему 1 и лемму 1. 
3.3. Комплекс Кошуля. Пусть $\mathfrak{g}$ - конечномерная алгебра Ли, $X$ - пространство Фреше, и пусть $\alpha: \mathfrak{g} \rightarrow \mathscr{L}(X)$ - представление Ли. Комплекс

$$
0 \leftarrow X \stackrel{d_{0}}{\longleftarrow} X \otimes \mathfrak{g} \stackrel{d_{1}}{\longleftarrow} \cdots \stackrel{d_{p-1}}{\longleftarrow} X \otimes \wedge^{p} \mathfrak{g} \stackrel{d_{p}}{\longleftarrow} \ldots
$$

называется комплексом Кошуля пары $(X, \alpha)$, где

$$
d_{p-1}(x \otimes \underline{u})=\sum_{i=1}^{p}(-1)^{i+1} \alpha\left(u_{i}\right) x \otimes \underline{u}_{i}+\sum_{i<j}(-1)^{i+j-1} x \otimes\left[u_{i}, u_{j}\right] \wedge \underline{u}_{i j}
$$

для $\underline{u}=u_{1} \wedge \cdots \wedge u_{p} \in \wedge^{p} \mathfrak{g} ;$ он обозначается через $\operatorname{Kos}(X, \alpha)$. Расширенный добавлением факторотображения $\pi: X \rightarrow X_{-1}$ комплекс Кошуля обозначается как $\operatorname{Kos}^{+}(X, \alpha)$, где $X_{-1}=X / \operatorname{im}\left(d_{0}\right)$.

Пусть $\mathfrak{g}$ является нильпотентной алгеброй Ли с треугольным базисом $e=$ $\left(e_{1}, \ldots, e_{n}\right)$. Положим $l_{i}=e_{n-i+1}, 1 \leqslant i \leqslant n$. Очевидно, что подпространство $I_{k}$ алгебры $\mathfrak{g}$, порожденное векторами $l_{1}, \ldots, l_{k}$, является идеалом Ли. Пусть $X-$ пространство Фреше, и пусть $\alpha: \mathfrak{g} \rightarrow \mathscr{L}(X)$ является представлением. Рассмотрим линейные операторы $T_{i} \in \mathscr{L}(X), T_{i} x=\alpha\left(l_{i}\right) x, 1 \leqslant i \leqslant n$.

Теорема 2. Если $T=\left(T_{1}, \ldots, T_{n}\right)$ является локально обратимым слева на $X$, mo $^{+} \operatorname{Kos}^{+}(X, \alpha)$ является допустимым цепным комплексом.

Этот результат был доказан в [24] (см. также [23]).

\section{§ 4. Оператор деления между банаховыми обертывающими алгебрами}

В этом параграфе мы докажем, что оператор деления на радикальную переменную, взятую из идеала $[\mathfrak{g}, \mathfrak{g}]$ алгебры Гейзенберга $\mathfrak{g}$, может быть расширен до ограниченного линейного оператора между некоторыми банаховыми обертывающими алгебрами алгебры Ли $\mathfrak{g}$.

4.1. Банахова обертывающая алгебра $\mathscr{A}(p)$. Пусть $\mathfrak{g}$ - конечномерная алгебра Ли, и пусть $p$ является нормой на $\mathfrak{g}$. Банахова алгебра $\mathscr{A}(p)$ и гомоморфизм $\varphi_{\mathfrak{g}}: \mathscr{U}(\mathfrak{g}) \rightarrow \mathscr{A}(p),\left\|\varphi_{\mathfrak{g}} \mid \mathfrak{g}\right\| \leqslant 1$, с плотным образом определяются единственным способом следующим универсально проективным свойством (см. [13], [14]).

Лемма 2. Если $\mathscr{B}$ является банаховой алгеброй $u \alpha: \mathfrak{g} \rightarrow \mathscr{B},\|\alpha\| \leqslant 1$,гомоморфизм Ли, то существует такой единственный гомоморфизм алгебр $\widetilde{\alpha}: \mathscr{A}(p) \rightarrow \mathscr{B},\|\widetilde{\alpha}\| \leqslant 1$, чmо $\widetilde{\alpha} \cdot \varphi_{\mathfrak{g}}=\alpha$.

СлеДСтвиЕ 2. Пусть $\mathfrak{g}$ - конечномерная алгебра Ли с нормой $p$. Существует единственный изометрический изоморфизм алгебр $\widetilde{\varkappa_{\mathfrak{g}}}: \mathscr{A}(p) \rightarrow \mathscr{A}(p)^{\mathrm{op}}$, расширяющий антипод $\varkappa_{\mathfrak{g}}: \mathscr{U}(\mathfrak{g}) \rightarrow \mathscr{U}(\mathfrak{g})^{\mathrm{op}}$, т.е. выполнено равенство

$$
\widetilde{\varkappa_{\mathfrak{g}}} \cdot \varphi_{\mathfrak{g}}=\varphi_{\mathfrak{g}} \cdot \varkappa_{\mathfrak{g}} .
$$


ДоказАтеЛЬСтво. Ясно, что $\rho: \mathscr{U}(\mathfrak{g}) \rightarrow \mathscr{A}(p)^{\mathrm{op}}, \rho=\varphi_{\mathfrak{g}} \cdot \varkappa_{\mathfrak{g}},-$ гомоморфизм алгебр. Более того,

$$
\|\rho(x)\|_{\mathscr{A}(p)}=\left\|-\varphi_{\mathfrak{g}}(x)\right\|_{\mathscr{A}(p)} \leqslant\left\|\varphi_{\mathfrak{g}}\right\| p(x) \leqslant p(x)
$$

для всех $x \in \mathfrak{g}$. Таким образом, $\left\|\left.\rho\right|_{\mathfrak{g}}\right\| \leqslant 1$. Существует гомоморфизм алгебр $\widetilde{\rho}: \mathscr{A}(p) \rightarrow \mathscr{A}(p)$ ор такой, что $\widetilde{\rho} \cdot \varphi_{\mathfrak{g}}=\rho$ и $\|\widetilde{\rho}\| \leqslant 1$ в силу леммы 2. Так как $\varkappa_{\mathfrak{g}}^{2}=1_{\mathscr{U}(\mathfrak{g})}$ и $\operatorname{im}\left(\varphi_{\mathfrak{g}}\right)$ порождает плотную подалгебру в $\mathscr{A}(p)$, то из этого вытекает, что $\widetilde{\rho}^{2}=1_{\mathscr{A}(p)}$. Следовательно, $\widetilde{\rho}$ является требуемой изометрией.

Следствие 2 доказано.

Алгебра $\mathscr{A}(p)$ называется (см. [14], [12]) банаховой обертывающей алгеброй банаховой алгебры Ли $\mathfrak{g}$, снабженной нормой $p$.

Пусть теперь $\mathfrak{g}$ - нильпотентная алгебра Ли с нормой $p$. В этом случае банахова обертывающая алгебра $\mathscr{A}(p)$ - пополнение по норме алгебры $\mathscr{U}(\mathfrak{g})$ (см. [13], [14]). Таким образом, $\mathscr{A}(p)$ является отмеченным пополнением алгебры $\mathscr{U}(\mathfrak{g})$ в силу леммы 2. Предположим, что $p=l_{e}$ является $\ell_{1}$-нормой (см. §2) в нильпотентной алгебре Ли $\mathfrak{g}$ относительно треугольного базиса $e=$ $\left(e_{1}, \ldots, e_{n}\right)$ в $\mathfrak{g}$. Семейство всех (неупорядоченных) мономов в $v=e_{i_{1}} \cdots e_{i_{s}} \in$ $\mathscr{U}(\mathfrak{g})$, взятое относительно базиса $e$, порождает $\mathscr{U}(\mathfrak{g})$ как линейное пространство, поэтому каждый элемент $u \in \mathscr{U}(\mathfrak{g})$ имеет разложение $u=a_{1} v_{1}+\cdots+a_{k} v_{k}$ в виде линейной комбинации этих мономов. Положим $\operatorname{deg}\left(e_{i}\right)=\max \{k$ : $\left.e_{i} \in \mathfrak{g}^{(k)}\right\}$ для всех элементов $e_{i}$ базиса $e$. Если $v=e_{i_{1}} \cdots e_{i_{s}}$, то $\operatorname{deg}(v)=$ $\operatorname{deg}\left(e_{i_{1}}\right)+\cdots+\operatorname{deg}\left(e_{i_{s}}\right)$. Для $u \in \mathscr{U}(\mathfrak{g})$ положим (см. [14])

$$
\|u\|=\inf \left\{\left|a_{1}\right|+\cdots+\left|a_{k}\right|: u=a_{1} v_{1}+\cdots+a_{k} v_{k}\right\}
$$

где наибольшая нижняя грань берется по всем разложениям элемента $u$. Так как $\mathfrak{g}$ является нильпотентной алгеброй Ли, то в (2) достаточно брать такие разложения элемента $u$ в качестве линейных комбинаций мономов, чьи степени нильпотентности ограничены сверху некоторой константой, зависящей от $u$ и $n$. Из этого следует, что $\|u\|=0$ тогда и только тогда, когда $u=0$. Более того, $\mathscr{U}(\mathfrak{g})$ является нормированной алгеброй относительно нормы $\|\cdot\|$. Если $\mathfrak{A}$ является ее пополнением по норме и $\varphi: \mathscr{U}(\mathfrak{g}) \rightarrow \mathfrak{A}$ есть каноническое вложение, то $(2)$ немедленно влечет, что пара $(\mathfrak{A}, \varphi)$ владеет универсально проективным свойством из леммы 2. Следовательно, $\mathfrak{A}=\mathscr{A}\left(l_{e}\right)$ с точностью до изометрического изоморфизма.

4.2. Алгебра Гейзенберга. Здесь будем считать, что g является алгеброй Гейзенберга с ее каноническим базисом $e=\left(e_{1}, e_{2}, e_{3}\right)$. Отметим, что все рассуждения, которые будут приведены ниже, остаются справедливыми для алгебры Гейзенберга произвольного размерности. Для краткости мы рассмотрим только случай трехмерной алгебры Гейзенберга и предположим, что $a=0$.

Пусть $\mathfrak{m}_{e} \subseteq \mathscr{U}(\mathfrak{g})$ есть подмножество всех (неупорядоченных) мономов $v=$ $e_{i_{1}} \cdots e_{i_{q}}$, взятых по базису $e$. Для $v=e_{i_{1}} \cdots e_{i_{q}} \in \mathfrak{m}_{e}$ и для набора $t \in \mathbb{R}_{+}^{3}$ мы положим $t^{v}=t_{i_{1}} \cdots t_{i_{q}}$. Говорят, что моном $v \in \mathfrak{m}_{e}$ является радикальным мономом, если $v=w e_{3}$ для некоторого $w \in \mathfrak{m}_{e}$. Более того, говорят, что 
радикальный моном является 1-радикальным мономом, если $v=w e_{3}$ и моном $w$ не содержит $e_{3}$. Предполагается, что длина $l(v)$ монома $v=e_{i_{1}} \cdots e_{i_{q}}$ равна $q$.

Возьмем моном $v \in \mathfrak{m}_{e}$. Путем перестановки базисных элементов $e_{i_{k}}$, возникающих в выражении от $v$, можно найти его разложение через упорядоченные мономы $e^{J}=e_{1}^{j_{1}} e_{2}^{j_{2}} e_{3}^{j_{3}} \in \mathfrak{m}_{e}$. Таким образом, если $v-$ нерадикальный моном, то существует определенный единственным образом упорядоченный моном $b(v) \in \mathfrak{m}_{e}$ такой, что

$$
v=b(v)+r(v),
$$

где $r(v)$ есть линейная комбинация некоторых 1-радикальных мономов $w_{k} \in \mathfrak{m}_{e}$, т.е. $r(v) \in \mathscr{U}(\mathfrak{g}) e_{3}$. В двух последующих леммах мы разберем детальное выражение для $r(v)$. Для удобства мы используем обозначения $x=e_{1}, y=e_{2}$, $z=e_{3}$. Очевидно, каждый нерадикальный моном $v \in \mathfrak{m}_{e}$ может быть записан как $v=x^{n_{1}} y^{k_{1}} \cdots x^{n_{j}} y^{k_{j}}$ для некоторых $n_{i}, k_{i} \geqslant 0$. Более того, $b(v)=$ $x^{n_{1}+\cdots+n_{j}} y^{k_{1}+\cdots+k_{j}}$ и $l(v)=\sum_{i}\left(n_{i}+k_{i}\right)$.

ЛЕмма 3. Пусть g является алгеброй Гейзенберга с треуголъным базисом $e=(x, y, z)$. Тогда

$$
\left[x^{n}, y^{k}\right]=k \sum_{i=1}^{n} x^{n-i} y^{k-1} x^{i-1} z .
$$

ДокАЗАТЕльство. Рассуждаем индукцией по $n$. Так как $\left[x, y^{k}\right]=k y^{k-1} z$, то формула немедленно выполнена для $n=1$. Пусть $n>1$. По предположению индукции мы получим, что

$$
\begin{aligned}
{\left[x^{n}, y^{k}\right] } & =x^{n-1}\left[x, y^{k}\right]+\left[x^{n-1}, y^{k}\right] x=k x^{n-1} y^{k-1} z+k \sum_{i=1}^{n-1} x^{n-i-1} y^{k-1} x^{i} z \\
& =k \sum_{i=1}^{n} x^{n-i} y^{k-1} x^{i-1} z,
\end{aligned}
$$

т.е. $\left[x^{n}, y^{k}\right]=k \sum_{i=1}^{n} x^{n-i} y^{k-1} x^{i-1} z$.

Лемма 3 доказана.

Лемма 4. Пусть $\mathfrak{g}$ есть алгебра Гейзенберга с ее треугольным базисом е = $(x, y, z)$, и псть $v=x^{n_{1}} y^{k_{1}} \cdots x^{n_{j}} y^{k_{j}} \in \mathfrak{m}_{e}$ является нерадикальным мономом. Тогда

$$
r(v)=-\sum_{p=1}^{j-1}\left(k_{1}+\cdots+k_{p}\right) \sum_{s=1}^{n_{p+1}} x^{n_{1}+\cdots+n_{p+1}-s} y^{k_{1}+\cdots+k_{p}-1} x^{s-1} y^{k_{p+1}} \cdots x^{n_{j}} y^{k_{j}} z
$$

m.е. $r(v)$ есть линейная комбинация 1-радикальных мономов, имеющих длину $l(v)-1$.

ДокАЗАТЕЛЬСтво. Применим индукцию по $j$. Для $j \leqslant 2$ формула следует из леммы 3.

Пусть теперь $w=x^{n_{1}} y^{k_{1}} \cdots x^{n_{j-1}} y^{k_{j-1}} \in \mathfrak{m}_{e}$. Тогда

$$
w=x^{n_{1}+\cdots+n_{j-1}} y^{k_{1}+\cdots+k_{j-1}}+r(w) .
$$


По предположению индукции имеем, что $r(w)=-\sum_{p=1}^{j-2}\left(k_{1}+\cdots+k_{p}\right) \sum_{s=1}^{n_{p+1}} x^{n_{1}+\cdots+n_{p+1}-s} y^{k_{1}+\cdots+k_{p}-1} x^{s-1} y^{k_{p+1}} \cdots x^{n_{j-1}} y^{k_{j-1}} z$.

Применяя опять лемму 3 , заключаем, что

$$
\begin{aligned}
v= & w x^{n_{j}} y^{k_{j}}=x^{n_{1}+\cdots+n_{j-1}} y^{k_{1}+\cdots+k_{j-1}} x^{n_{j}} y^{k_{j}}+r(w) x^{n_{j}} y^{k_{j}} \\
= & x^{n_{1}+\cdots+n_{j}} y^{k_{1}+\cdots+k_{j}} \\
& \quad-\left(k_{1}+\cdots+k_{j-1}\right) \sum_{s=1}^{n_{j}} x^{n_{1}+\cdots+n_{j-1}+n_{j}-s} y^{k_{1}+\cdots+k_{j-1}-1} x^{s-1} y^{k_{j}} z \\
& \quad-\sum_{p=1}^{j-2}\left(k_{1}+\cdots+k_{p}\right) \sum_{s=1}^{n_{p+1}} x^{n_{1}+\cdots+n_{p+1}-s} y^{k_{1}+\cdots+k_{p}-1} x^{s-1} y^{k_{p+1}} \cdots x^{n_{j}} y^{k_{j}} z \\
= & b(v)-\sum_{p=1}^{j-1}\left(k_{1}+\cdots+k_{p}\right) \sum_{s=1}^{n_{p+1}} x^{n_{1}+\cdots+n_{p+1}-s} y^{k_{1}+\cdots+k_{p}-1} x^{s-1} y^{k_{p+1}} \cdots x^{n_{j}} y^{k_{j}} z .
\end{aligned}
$$

Таким образом, требуемый вид разложения $r(v)$ получен.

Лемма 4 доказана.

4.3. Оператор деления. Возьмем $s, t \in \mathbb{R}_{+}^{3}, t<s$. Напомним (см. (2)), что норма $\|u\|_{\mathscr{A}\left(l_{e, t}\right)}$ полинома $u \in \mathscr{U}(\mathfrak{g})$ в банаховой обертывающей алгебре $\mathscr{A}\left(l_{e, t}\right)$ (см. п. 4.1) вычисляется по правилу

$$
\|u\|_{\mathscr{A}\left(l_{e, t}\right)}=\inf \left\{\sum_{i=1}^{m}\left|a_{i}\right|: u=\sum_{i=1}^{m} a_{i}\left(t^{-1}\right)^{v_{i}} v_{i}\right\}
$$

где наибольшая нижняя грань берется по всем представлениям от $u$ как линейной комбинации мономов $\left(t^{-1}\right)^{v_{i}} v_{i} \in \mathfrak{m}_{t^{-1}}$, взятых по базису $t^{-1} e$, где $v_{i} \in \mathfrak{m}_{e}$.

Снова предположим, что $\mathfrak{g}$ является алгеброй Гейзенберга с треугольным базисом $e=(x, y, z)$, и рассмотрим оператор деления

$$
\nabla_{z} \in \mathscr{L}(\mathscr{U}(\mathfrak{g})), \quad \nabla_{z}\left(x^{n} y^{m} z^{k}\right)=\delta_{k} x^{n} y^{m} z^{k-1}
$$

(cм. §2).

Лемма 5. Пусть $u \in \mathscr{U}(\mathfrak{g}) z, u$ nусть $s, t \in \mathbb{R}_{+}^{3}$. Тогда

$$
\left\|\nabla_{z} u\right\|_{\mathscr{A}\left(l_{e, t}\right)}=s_{3}^{-1} \inf \left\{\sum_{i}\left|b_{i}\right|\left(s^{-1} t\right)^{v_{i}}: u=\sum_{i} b_{i}\left(s^{-1}\right)^{v_{i} z} v_{i} z\right\}
$$

где наибольшая нижняя грань берется по всем представлениям от и в виде линейной комбинации радикальнъх мономов $\left(s^{-1}\right)^{v_{i} z} v_{i} z \in \mathfrak{m}_{s^{-1}}$.

ДокАЗАтЕльство. Сначала отметим, что если $v=w z\left(w \in \mathfrak{m}_{e}\right)$ является радикальным мономом и $w=\sum_{i} a_{i} v_{i}$ является разложением $w$ посредством упорядоченных мономов $v_{i} \in \mathfrak{m}_{e}$, то $v=\sum_{i} a_{i} v_{i} z$ является разложением такого 
же вида для $v$ и $\nabla_{z} v=\nabla_{z} \sum_{i} a_{i} v_{i} z=\sum_{i} a_{i} v_{i}=w$. Таким образом, если $u=$ $\sum_{i} b_{i}\left(s^{-1}\right)^{v_{i} z} v_{i} z\left(v_{i} \in \mathfrak{m}_{e}\right)$ является разложением $u$ посредством радикальных мономов из $\mathfrak{m}_{s^{-1}}$, то

$$
\nabla_{z} u=s_{3}^{-1} \sum_{i} b_{i}\left(s^{-1}\right)^{v_{i}} v_{i}=s_{3}^{-1} \sum_{i}\left(s^{-1} t\right)^{v_{i}} b_{i}\left(t^{-1}\right)^{v_{i}} v_{i} .
$$

Из этого вытекает, что $\left\|\nabla_{z} u\right\|_{\mathscr{A}\left(l_{e, t}\right)} \leqslant s_{3}^{-1} \sum_{i}\left|b_{i}\right|\left(s^{-1} t\right)^{v_{i}}$.

Обратно, если $\nabla_{z} u=\sum_{j} a_{j}\left(t^{-1}\right)^{v_{j}} v_{j}$ для некоторого $v_{j} \in \mathfrak{m}_{e}$, то, принимая во внимание, что $u \in \mathscr{U}(\mathfrak{g}) z$ и $R_{z} \nabla_{z}=1$ в $\mathscr{U}(\mathfrak{g}) z$, заключаем, что

$$
u=\sum_{j} a_{j}\left(t^{-1}\right)^{v_{j}} v_{j} z=\sum_{j} b_{j}\left(s^{-1}\right)^{v_{j} z} v_{j} z
$$

где $b_{j}=s_{3} a_{j}\left(s t^{-1}\right)^{v_{j}}$. Из этого следует, что

$$
\left\|\nabla_{z} u\right\|_{\mathscr{A}\left(l_{e, t}\right)} \leqslant \sum_{j}\left|a_{j}\right|=\sum_{j} s_{3}^{-1}\left|b_{j}\right|\left(s^{-1} t\right)^{v_{j}} .
$$

Таким образом,

$$
\left\|\nabla_{z} u\right\|_{\mathscr{A}\left(l_{e, t}\right)}=s_{3}^{-1} \inf \left\{\sum_{i}\left|b_{i}\right|\left(s^{-1} t\right)^{v_{i}}: u=\sum_{i} b_{i}\left(s^{-1}\right)^{v_{i} z} v_{i} z\right\}
$$

в силу (3).

Лемма 5 доказана.

Теперь возьмем $u \in \mathscr{U}(\mathfrak{g}) z$, и пусть $u=\sum_{m} \lambda_{m}\left(s^{-1}\right)^{v_{m}} v_{m}$ - его разложение, в котором $v_{m} \in \mathfrak{m}_{e}$. Разделим последнюю сумму на две части:

$$
u=\sum_{\rho} \lambda_{\rho}\left(s^{-1}\right)^{v_{\rho}} v_{\rho}+\sum_{j} \lambda_{j}\left(s^{-1}\right)^{w_{j} z} w_{j} z
$$

где $\left\{v_{\rho}\right\}$ являются нерадикальными мономами, a $\left\{w_{j} z\right\}$ - радикальными. Поскольку $v_{\rho}=b\left(v_{\rho}\right)+r\left(v_{\rho}\right)$, из этого следует, что

$$
u=\sum_{\rho} \lambda_{\rho}\left(s^{-1}\right)^{v_{\rho}} b\left(v_{\rho}\right)+\sum_{\rho} \lambda_{\rho}\left(s^{-1}\right)^{v_{\rho}} r\left(v_{\rho}\right)+\sum_{j} \lambda_{j}\left(s^{-1}\right)^{w_{j} z} w_{j} z .
$$

Принимая во внимание, что $u \in \mathscr{U}(\mathfrak{g}) z$ и $\mathscr{U}(\mathfrak{g})=\operatorname{ker}\left(\nabla_{z}\right) \oplus \mathscr{U}(\mathfrak{g}) z($ см. лемму 1$)$, получим, что $\sum_{\rho} \lambda_{\rho}\left(s^{-1}\right)^{v_{\rho}} b\left(v_{\rho}\right)=0$. Таким образом, мы получим разложение

$$
u=\sum_{\rho} \lambda_{\rho}\left(s^{-1}\right)^{v_{\rho}} r\left(v_{\rho}\right)+\sum_{j} \lambda_{j}\left(s^{-1}\right)^{w_{j} z} w_{j} z
$$

элемента $u$ посредством радикальных мономов из $\mathfrak{m}_{s^{-1}}$.

Лемма 6. Пусть $\mathfrak{g}$ - алгебра Гейзенберга с треугольным базисом $e=(x$, $y, z)$. Оператор деления $\nabla_{z} \in \mathscr{L}(\mathscr{U}(\mathfrak{g}))$ имеет расширение до ограниченного линейного оператора $\nabla_{z}: \mathscr{A}\left(l_{e, s}\right) \rightarrow \mathscr{A}\left(l_{e, t}\right)$ при условии, что $s, t \in \mathbb{R}_{+}^{3} u t<s$. 
ДоказАтельство. Отметим, что радикал Джекобсона $\operatorname{Rad} \mathscr{A}\left(l_{e, s}\right)$ является замыканием $\mathscr{U}(\mathfrak{g}) z$ и $\mathscr{A}\left(l_{e, s}\right)=\mathscr{S}_{s} \oplus \operatorname{Rad} \mathscr{A}\left(l_{e, s}\right)$, где $\mathscr{S}_{s}$ является замкнутым подпространством, содержащим все абсолютно сходящиеся степенные ряды $\sum_{n, m} a_{n m} x^{n} y^{m}$ в $\mathscr{A}\left(l_{e, s}\right)$ (см. [14]). Поэтому достаточно доказать, что суженный оператор $\nabla_{z}: \mathscr{U}(\mathfrak{g}) z \rightarrow \mathscr{A}\left(l_{e, t}\right)$ непрерывен в топологии нормы пространства $\mathscr{A}\left(l_{e, s}\right)$. Пусть

$$
\delta=\max \left(s^{-1} t\right), \quad C_{s}=\max \left\{\left(s_{1} s_{2}\right)^{-1}, s_{3}^{-1}\right\}
$$

и возьмем $u \in \mathscr{U}(\mathfrak{g}) z$. Для зафиксированного $\varepsilon>0$ можно выбрать разложение

$$
u=\sum_{\rho} \lambda_{\rho}\left(s^{-1}\right)^{v_{\rho}} v_{\rho}+\sum_{j} \lambda_{j}\left(s^{-1}\right)^{w_{j} z} w_{j} z
$$

такое, что $\sum_{\rho}\left|\lambda_{\rho}\right|+\sum_{j}\left|\lambda_{j}\right|<\|u\|_{\mathscr{A}\left(l_{e, s}\right)}+\varepsilon$, где $\left\{v_{\rho}\right\}$ - нерадикальные мономы. Из этого вытекает, что

$$
u=\sum_{\rho} \lambda_{\rho}\left(s^{-1}\right)^{v_{\rho}} r\left(v_{\rho}\right)+\sum_{j} \lambda_{j}\left(s^{-1}\right)^{w_{j} z} w_{j} z
$$

в силу (4). Пусть $v_{\rho}=x^{n_{1}^{(\rho)}} y^{m_{1}^{(\rho)}} \cdots x^{n_{j_{\rho}}^{(\rho)}} y^{m_{j_{\rho}}^{(\rho)}}$, поэтому $l\left(v_{\rho}\right)=\sum_{i=1}^{j_{\rho}}\left(n_{i}^{(\rho)}+m_{i}^{(\rho)}\right)$. Применяя лемму 4, выводим, что

$$
\left(s^{-1}\right)^{v_{\rho}} r\left(v_{\rho}\right)=-\left(s_{1} s_{2}\right)^{-1} s_{3} \sum_{q=1}^{j_{\rho}-1}\left(m_{1}^{(\rho)}+\cdots+m_{q}^{(\rho)}\right) \sum_{k=1}^{n_{q+1}^{(\rho)}}\left(s^{-1}\right)^{w_{\rho q k} z} w_{\rho q k} z
$$

где

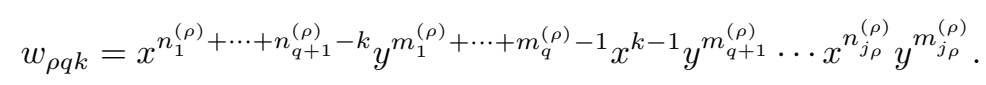

Тогда

$$
\begin{aligned}
u=- & \left(s_{1} s_{2}\right)^{-1} s_{3} \sum_{\rho} \sum_{q=1}^{j_{\rho}-1} \lambda_{\rho}\left(m_{1}^{(\rho)}+\cdots+m_{q}^{(\rho)}\right) \sum_{k=1}^{n_{q+1}^{(\rho)}}\left(s^{-1}\right)^{w_{\rho q k} z} w_{\rho q k} z \\
& +\sum_{j} \lambda_{j}\left(s^{-1}\right)^{w_{j} z} w_{j} z
\end{aligned}
$$

является разложением элемента $u$ посредством радикальных мономов из $\mathfrak{m}_{s^{-1}}$. Применяя лемму 5, мы получим, что

$$
\begin{aligned}
& \left\|\nabla_{z} u\right\|_{\mathscr{A}\left(l_{e, t}\right)} \leqslant\left(s_{1} s_{2}\right)^{-1} \sum_{\rho}\left|\lambda_{\rho}\right| \sum_{q=1}^{j_{\rho}-1}\left(m_{1}^{(\rho)}+\cdots+m_{q}^{(\rho)}\right) \sum_{k=1}^{n_{q+1}^{(\rho)}}\left(s^{-1} t\right)^{w_{\rho q k}} \\
& \quad+s_{3}^{-1} \sum_{j}\left|\lambda_{j}\right|\left(s^{-1} t\right)^{w_{j}} \\
& \leqslant C_{s}\left(\sum_{\rho}\left|\lambda_{\rho}\right| \sum_{q=1}^{j_{\rho}-1}\left(m_{1}^{(\rho)}+\cdots+m_{q}^{(\rho)}\right) n_{q+1}^{(\rho)} \delta^{l\left(v_{\rho}\right)-2}+\sum_{j}\left|\lambda_{j}\right| \delta^{l\left(w_{j}\right)}\right) \\
& \leqslant C_{s}\left(\sum_{\rho}\left|\lambda_{\rho}\right| \sum_{q=1}^{j_{\rho}-1}\left(\sum_{i=1}^{q} m_{i}^{(\rho)}\right) \delta^{\sum_{i=1}^{q} m_{i}^{(\rho)}-1}\left(\sum_{i=1}^{q+1} n_{i}^{(\rho)}\right) \delta^{\sum_{i=1}^{q+1} n_{i}^{(\rho)}-1}+\sum_{j}\left|\lambda_{j}\right|\right)
\end{aligned}
$$




$$
\begin{aligned}
& \leqslant C_{s}\left(\sum_{\rho}\left|\lambda_{\rho}\right|\left(\sum_{k=1}^{\infty} k \delta^{k-1}\right)^{2}+\sum_{j}\left|\lambda_{j}\right|\right) \\
& \leqslant C_{s}(1-\delta)^{-4}\left(\sum_{\rho}\left|\lambda_{\rho}\right|+\sum_{j}\left|\lambda_{j}\right|\right) \leqslant C_{s}(1-\delta)^{-4}\left(\|u\|_{\mathscr{A}\left(l_{e, s}\right)}+\varepsilon\right),
\end{aligned}
$$

т.е. $\left\|\nabla_{z} u\right\|_{\mathscr{A}\left(l_{e, t}\right)} \leqslant C_{s}(1-\delta)^{-4}\left(\|u\|_{\mathscr{A}\left(l_{e, s}\right)}+\varepsilon\right)$. Так как $\varepsilon$ было произвольным, то из этого вытекает, что

$$
\left\|\nabla_{z} u\right\|_{\mathscr{A}\left(l_{e, t}\right)} \leqslant C_{s}(1-\delta)^{-4}\|u\|_{\mathscr{A}\left(l_{e, s}\right)}
$$

Отсюда получаем, что $\nabla_{z}$ есть ограниченный оператор.

Лемма 6 доказана.

Оценка (5), предложенная в доказательстве леммы 6, позволяет выяснить скорость сходимости $\left\|z^{n}\right\|_{\mathscr{A}\left(l_{e}\right)}^{1 / n}$. Ранее мы уже отмечали, что $\left\|z^{n}\right\|_{\mathscr{A}\left(l_{e}\right)}^{1 / n}=O\left(n^{-1}\right)$ (см. (1)).

СлеДСтвиЕ 3. Для достаточно больших п справедлива ощенка

$$
n^{-4} \leqslant\left\|z^{n}\right\|_{\mathscr{A}\left(l_{e}\right)}^{1 / n} \leqslant 2 n^{-1} .
$$

ДокАЗАТЕЛЬство. Так как $\|x\|_{\mathscr{A}\left(l_{e}\right)} \leqslant 1$ и $\|y\|_{\mathscr{A}\left(l_{e}\right)} \leqslant 1$, то из этого следует, что $\left\|z^{n}\right\|_{\mathscr{A}\left(l_{e}\right)}^{1 / n} \leqslant 2 n^{-1}$ в силу (1). Далее, зафиксируем набор трех чисел $t=(t, t, t)$, где $0<t<1$, и пусть $t^{k}=\left(t^{k}, t^{k}, t^{k}\right), k \in \mathbb{N}$. Тогда $\left\{t^{k}: k \in \mathbb{N}\right\}-$ убывающая последовательность в $\mathbb{R}_{+}^{3}$. Существуют ограниченные линейные операторы деления $\nabla_{z}: \mathscr{A}\left(l_{e, t^{k}}\right) \rightarrow \mathscr{A}\left(l_{e, t^{k+1}}\right)$ и $\left\|\nabla_{z}\right\| \leqslant t^{-2 k}(1-t)^{-4}$ в силу (5) для любого $k \in \mathbb{N}$. Применяя операторы $\nabla_{z}$ к $z^{n} \in \mathscr{A}\left(l_{e}\right)$, получим, что $\nabla_{z}^{n} z^{n}=1_{\mathscr{A}\left(l_{e, t^{n}}\right)}$. Из этого вытекает, что $1 \leqslant t^{-n(n-1)}(1-t)^{-4 n}\left\|z^{n}\right\|_{\mathscr{A}\left(l_{e}\right)}$, откуда $\left\|z^{n}\right\|_{\mathscr{A}\left(l_{e}\right)}^{1 / n} \geqslant t^{n-1}(1-t)^{4}$ для всех $t<1$. Остается отметить, что

$$
\max \left\{\varphi_{n}(t): 0 \leqslant t \leqslant 1\right\}=\varphi_{n}\left(\frac{n-1}{n+3}\right),
$$

где $\varphi_{n}(t)=t^{n-1}(1-t)^{4}$. Таким образом, $\left\|z^{n}\right\|_{\mathscr{A}\left(l_{e}\right)}^{1 / n} \geqslant n^{-4}$ для достаточно больших $n$.

Следстве 3 доказано.

\section{§ 5. Некоммутативные голоморфные функции}

В этом параграфе мы докажем, что алгебра Фреше-Аренса-Майкла некоммутативных голоморфных функций в полидиске от элементов алгебры Гейзенберга $\mathfrak{g}$ является слабо обращающим пополнением алгебры $\mathscr{U}(\mathfrak{g})$.

5.1. Алгебры Фреше $\mathscr{O}_{\mathfrak{g}}\left(D_{r}\right)$. Пусть $\mathfrak{g}$ - конечномерная алгебра Ли, и пусть $e=\left(e_{1}, \ldots, e_{n}\right)$ является его базисом таким, что $\left(e_{m+1}, \ldots, e_{n}\right)$ - базис в $[\mathfrak{g}, \mathfrak{g}]$ для некоторого $m$. Пространство всех характеров Ли $\Delta(\mathfrak{g})$ отождествляется с пространством $\mathbb{C}^{m}$ посредством отображения

$$
\Delta(\mathfrak{g}) \rightarrow \mathbb{C}^{m}, \quad a \mapsto\left(a\left(e_{1}\right), \ldots, a\left(e_{m}\right)\right),
$$


где $m=\operatorname{dim}\left(\mathfrak{g} / \mathfrak{g}^{(2)}\right)$. Рассмотрим полидиск $D_{r}$ в $\mathbb{C}^{n}$ мультирадиуса $r=\left(r_{1}\right.$, $\left.\ldots, r_{n}\right) \in \overline{\mathbb{R}}_{+}^{n}$ с центром в нуле. Алгебра $\mathscr{O}_{\mathfrak{g}}\left(D_{r}\right)$ всех голоморфных функций от элементов алгебры Ли $\mathfrak{g}$ определяется (см. [13], [14]) как хаусдорфово пополнение Фреше универсальной обертывающей алгебры $\mathscr{U}(\mathfrak{g})$ в топологии, порожденной семейством полунорм $\left\{\|\cdot\|_{\mathscr{A}\left(l_{e, s}\right)}: s<r\right\}$, где $\|\cdot\|_{\mathscr{A}\left(l_{e, s}\right)}-$ норма в $\mathscr{U}(\mathfrak{g})$, унаследованная от банаховой обертывающей алгебры $\mathscr{A}\left(l_{e, s}\right)$ (см. п. 4.1), построенной по $\ell_{1}$-норме $l_{e, s}$ в $\mathfrak{g}$. Если $r=\infty$ (т.е. все $\left.r_{i}=+\infty\right)$, то $\mathscr{O}_{\mathfrak{g}}\left(\mathbb{C}^{n}\right)=\mathscr{O}_{\mathfrak{g}}$ называется алгеброй всех цельх функиий от элементов базиса е. В [13] доказано, что в случае, когда $\mathfrak{g}$ является разрешимой алгеброй Ли, пространство всех непрерывных характеров на $\mathscr{O}_{\mathfrak{g}}\left(D_{r}\right)$ отождествляется с суженным полидиском $D_{r_{\mathrm{s}}} \subseteq \mathbb{C}^{m}=\Delta(\mathfrak{g})$, где $r_{\mathrm{s}}=\left(r_{1}, \ldots, r_{m}\right), m=\operatorname{dim}\left(\mathfrak{g} / \mathfrak{g}^{(2)}\right)$. Более того, $\mathscr{O}_{\mathfrak{g}}\left(D_{r}\right)=\mathscr{O}_{\mathfrak{g}}\left(D_{t}\right)$ с точностью до топологического изоморфизма алгебр при условии $r_{\mathrm{s}}=t_{\mathrm{s}}$ (см. [14]). Поэтому алгебра $\mathscr{O}_{\mathfrak{g}}\left(D_{r}\right)$ не зависит от радикальной части $r_{m+1}, \ldots, r_{n}$ и полностью определена полупростой частью $r_{\mathrm{s}}$. В работе [14] также доказано, что факторалгебра $\mathscr{O}_{\mathfrak{g}}\left(D_{r}\right) / \operatorname{Rad} \mathscr{O}_{\mathfrak{g}}\left(D_{r}\right)$ по модулю ее радикала Джекобсона топологически изоморфна алгебре $\mathscr{O}\left(D_{r_{\mathrm{s}}}\right)$ всех обычных голоморфных функций в полидиске $D_{r_{\mathrm{s}}} \subseteq \Delta(\mathfrak{g})$. Напомним, что алгебра $\mathscr{O}_{\mathfrak{g}}\left(D_{r}\right)$ является алгеброй Аренса-Майкла, получаемой хаусдорфовым пополнением алгебры $\mathscr{U}(\mathfrak{g})$ по мультипликативному семейству полунорм $\left\{\|\cdot\|_{\mathscr{A}\left(l_{e, s}\right)}: s<r\right\}$ (см. п. 4.1). В частности, $\mathscr{O}_{\mathfrak{g}}\left(D_{r}\right)$ является алгеброй Фреше-Аренса-Майкла и антипод $\varkappa_{\mathfrak{g}}: \mathscr{U}(\mathfrak{g}) \rightarrow \mathscr{U}(\mathfrak{g})^{\text {op }}$ расширяется до топологического изоморфизма $\mathscr{O}_{\mathfrak{g}}\left(D_{r}\right) \rightarrow \mathscr{O}_{\mathfrak{g}}\left(D_{r}\right)^{\text {op }}$ алгебр Фреше в силу следствия 2.

Важным свойством алгебры $\mathscr{O}_{\mathfrak{g}}$ всех целых функций является то обстоятельство, что она является оболочкой Аренса-Майкла (см. [16; п. 5.2.21]) алгебры $\mathscr{U}(\mathfrak{g})$. А именно, каждый гомоморфизм банаховой алгебры $\mathscr{U}(\mathfrak{g}) \rightarrow A$ может быть поднят до единственного непрерывного гомоморфизма алгебры $\mathscr{O}_{\mathfrak{g}} \rightarrow A$ (см. [14]). В частности, если $X$ - левый банахов $\mathscr{U}(\mathfrak{g})$-модуль, то он имеет единственную структуру левого банахова $\mathscr{O}_{\mathfrak{g}}$-модуля, которая расширяет свою первоначальную $\mathscr{U}(\mathfrak{g})$-модульную структуру.

Возьмем точку $a \in \Delta(\mathfrak{g})$, и пусть $\mathfrak{g}-a$ является подалгеброй Ли в $\mathscr{U}(\mathfrak{g})$, составленной из элементов $u-a(u), u \in \mathfrak{g}$. Положим $\mathscr{O}_{\mathfrak{g}}\left(D_{a, r}\right)=\mathscr{O}_{\mathfrak{g}-a}\left(D_{r}\right)$ для полидиска $D_{a, r} \subseteq \mathbb{C}^{n}$ мультирадиуса $r$ с центром в точке $a$. Заметим, что центральная точка $a$ отождествляется с тривиальным характером $\varepsilon_{\mathfrak{g}-a}$, т.е. алгебра $\mathscr{O}_{\mathfrak{g}}\left(D_{a, r}\right)$ является отмеченной алгеброй с непрерывным характером $a: \mathscr{O}_{\mathfrak{g}}\left(D_{a, r}\right) \rightarrow \mathbb{C}$. Если $e-$ треугольный базис нильпотентной алгебры Ли $\mathfrak{g}$ и $\mathscr{S}_{e}\left(D_{a, r}\right)$ является подпространством в $\mathscr{O}_{\mathfrak{g}}\left(D_{a, r}\right)$ всех абсолютно сходящихся степенных рядов $\sum_{J} x_{J}\left(e_{1}-a_{1}\right)^{j_{1}} \cdots\left(e_{m}-a_{m}\right)^{j_{m}}$, то подпространство $\mathscr{S}_{e}\left(D_{a, r}\right)$ замкнуто,

$$
\mathscr{O}_{\mathfrak{g}}\left(D_{a, r}\right)=\mathscr{S}_{e}\left(D_{a, r}\right) \oplus \operatorname{Rad} \mathscr{O}_{\mathfrak{g}}\left(D_{a, r}\right)
$$

и

$$
\mathscr{S}_{e}\left(D_{a, r}\right)=\mathscr{O}\left(D_{a, r_{\mathrm{s}}}\right)
$$

с точностью до изоморфизма пространств Фреше (см. [17], [14]). Таким образом, факторгомоморфизм (взятый по модулю $\left.\operatorname{Rad} \mathscr{O}_{\mathfrak{g}}\left(D_{a, r}\right)\right)$

$$
\tau_{a, r}: \mathscr{O}_{\mathfrak{g}}\left(D_{a, r}\right) \rightarrow \mathscr{O}\left(D_{a, r_{\mathrm{s}}}\right)
$$


является ретракцией с правым обратным $\epsilon_{a, r}: \mathscr{O}\left(D_{a, r_{\mathrm{s}}}\right) \rightarrow \mathscr{S}_{e}\left(D_{a, r}\right)$,

$$
\epsilon_{a, r}\left(z_{1}-a_{1}\right)^{j_{1}} \cdots\left(z_{m}-a_{m}\right)^{j_{m}}=\left(e_{1}-a_{1}\right)^{j_{1}} \cdots\left(e_{m}-a_{m}\right)^{j_{m}} .
$$

5.2. Алгебра $\mathscr{O}_{\mathfrak{g}}\left(D_{r}\right)$ является слабо обращающим пополнением $\mathscr{U}(\mathfrak{g})$. Теперь предположим, что $D_{r} \subseteq \mathbb{C}^{3}$ - полидиск мультирадиуса $r=$ $\left(r_{1}, r_{2}, r_{3}\right)$ с центром в нулевой точке. Поскольку $z$ - элемент центра алгебры $\mathscr{O}_{\mathfrak{g}}\left(D_{r}\right)$, получается, что $\operatorname{im}\left(R_{z}\right)=\mathscr{O}_{\mathfrak{g}}\left(D_{r}\right) z$ - главный идеал в $\mathscr{O}_{\mathfrak{g}}\left(D_{r}\right)$.

ПреДЛОжЕНИЕ 1. Пусть $\mathfrak{g}$ является алгеброй Гейзенберга. Тогда оператор деления $\nabla_{z} \in \mathscr{L}(\mathscr{U}(\mathfrak{g}))$ имеет непрерывное расширение до оператора $\nabla_{z} \in$ $\mathscr{L}\left(\mathscr{O}_{\mathfrak{g}}\left(D_{r}\right)\right)$. В частности, оператор $R_{z} \in \mathscr{L}\left(\mathscr{O}_{\mathfrak{g}}\left(D_{r}\right)\right)$ является коректракиией с левым обратным $\nabla_{z}$ и

$$
\operatorname{Rad} \mathscr{O}_{\mathfrak{g}}\left(D_{r}\right)=\mathscr{O}_{\mathfrak{g}}\left(D_{r}\right) z
$$

ДоказАтельство. Достаточно доказать, что оператор $\nabla_{z} \in \mathscr{L}(\mathscr{U}(\mathfrak{g}))$ непрерывен в топологии алгебры $\mathscr{U}(\mathfrak{g})$, порожденной семейством норм $\left\{\|\cdot\|_{\mathscr{A}\left(l_{e, s}\right)}\right.$ : $s<r\}$. Зафиксируем набор $t \in \mathbb{R}_{+}^{3}$ такой, что $t<r$. Тогда $t<s<r$ для некоторого $s \in \mathbb{R}_{+}^{3}$. По лемме 6 оператор $\nabla_{z} \in \mathscr{L}(\mathscr{U}(\mathfrak{g}))$ имеет расширение до ограниченного линейного оператора $\nabla_{z}: \mathscr{A}\left(l_{e, s}\right) \rightarrow \mathscr{A}\left(l_{e, t}\right)$. Это означает, что $\nabla_{z} \in \mathscr{L}(\mathscr{U}(\mathfrak{g}))$ непрерывен относительно семейства норм $\left\{\|\cdot\|_{\mathscr{A}\left(l_{e, s}\right)}\right.$ : $s<r\}$. Поскольку $\nabla_{z} R_{z}=1$ в $\mathscr{U}(\mathfrak{g})$, получим, что $\nabla_{z}$ - левый обратный оператора $R_{z}$, действующего в $\mathscr{O}_{\mathfrak{g}}\left(D_{r}\right)$. В частности, $\operatorname{im}\left(R_{z}\right)$ замкнут. Так как $\operatorname{Rad} \mathscr{O}_{\mathfrak{g}}\left(D_{r}\right)$ является замкнутым идеалом, порожденным $z$ (см. [14]), то получаем $\operatorname{Rad} \mathscr{O}_{\mathfrak{g}}\left(D_{r}\right)=\mathscr{O}_{\mathfrak{g}}\left(D_{r}\right) z$.

Предложение 1 доказано.

ТеОРема 3. Пусть $\mathfrak{g}$ - алгебра Гейзенберга. Тогда $\mathscr{O}_{\mathfrak{g}}\left(D_{r}\right)$ является слабо обращающим пополнением алгебры $\mathscr{U}(\mathfrak{g})$.

ДокАзАтельство. Сначала отметим, что $\mathscr{O}_{\mathfrak{g}}\left(D_{r}\right)=\mathscr{S}_{e}\left(D_{r}\right) \oplus \operatorname{Rad} \mathscr{O}_{\mathfrak{g}}\left(D_{r}\right)$ в силу $(6)$, где $\mathscr{S}_{e}\left(D_{r}\right)$ является подпространством в $\mathscr{O}_{\mathfrak{g}}\left(D_{r}\right)$, составленным из всех абсолютно сходящихся степенных рядов $\sum a_{n m} x^{n} y^{m}$. Более того, каждый ряд

$$
f=\sum a_{n m} x^{n} y^{m} \in \mathscr{S}_{e}\left(D_{r}\right)
$$

определяет обычную голоморфную функцию

$$
\widehat{f}\left(w_{1}, w_{2}\right)=\tau_{0, r}(f)=\sum a_{n m} w_{1}^{n} w_{2}^{m}
$$

(от двух комплексных переменных $w_{1}$ и $w_{2}$ ), являющуюся элементом алгебры $\mathscr{O}\left(D_{r_{s}}\right)$, где $r_{s}=\left(r_{1}, r_{2}\right)$. Более того, правило $f \mapsto \widehat{f}$ осуществляет топологический изоморфизм пространств $Ф$ реше $\mathscr{S}_{e}\left(D_{r}\right)$ и $\mathscr{O}\left(D_{r_{s}}\right)$ (см. п. 5.1).

На основании следствия 1 мы должны доказать, что все операторы $\nabla_{e_{i}} \in$ $\mathscr{L}\left(\mathscr{U}_{i}^{e}\right), 1 \leqslant i \leqslant 3$, непрерывны (относительно топологии, унаследованной от $\left.\mathscr{O}_{\mathfrak{g}}\left(D_{r}\right)\right)$. Но $\mathscr{U}_{3}^{e}=\mathscr{U}(\mathfrak{g})$ и $\nabla_{e_{3}}$ (отметим, что $\nabla_{e_{3}}=\nabla_{z}$ ) есть непрерывный оператор в силу предложения 1 . Остается доказать непрерывность операторов $\nabla_{e_{i}} \in \mathscr{L}\left(\mathscr{U}_{i}^{e}\right), i=1,2$. Для этого применим разложение

$$
\mathscr{O}_{\mathfrak{g}}\left(D_{r}\right)=\mathscr{S}_{e}\left(D_{r}\right) \oplus \operatorname{Rad} \mathscr{O}_{\mathfrak{g}}\left(D_{r}\right)
$$


По определению $\mathscr{U}_{1}^{e} \subseteq \mathscr{U}_{2}^{e} \subseteq \mathscr{S}_{e}\left(D_{r}\right)$, поэтому они отождествляются с подпространствами в $\mathscr{O}\left(D_{r_{s}}\right)$. Легко проверить, что $\mathscr{U}_{2}^{e}$ сводится к коммутативной алгебре всех полиномов $\mathscr{P}\left(w_{1}, w_{2}\right)$ от двух комплексных переменных $w_{1}$ и $w_{2}$, а $\mathscr{U}_{1}^{e}-$ к подалгебре $\mathscr{P}\left(w_{1}\right)$ в $\mathscr{P}\left(w_{1}, w_{2}\right)$, порожденной $w_{1}$. Более того, операторы деления $\nabla_{x} \in \mathscr{L}\left(\mathscr{U}_{1}^{e}\right)$ и $\nabla_{y} \in \mathscr{L}\left(\mathscr{U}_{2}^{e}\right)$ сводятся к операторам того же типа $\nabla_{w_{i}} \in \mathscr{L}\left(\mathscr{P}\left(w_{1}, w_{2}\right)\right), i=1,2$, которые имеют расширения по непрерывности до операторов на $\mathscr{O}\left(D_{r_{s}}\right)$. В самом деле, достаточно положить

$$
\begin{aligned}
& \left(\nabla_{w_{1}} f\right)\left(w_{1}, w_{2}\right)=w_{1}^{-1}\left(f\left(w_{1}, w_{2}\right)-f\left(0, w_{2}\right)\right), \\
& \left(\nabla_{w_{2}} f\right)\left(w_{1}, w_{2}\right)=w_{2}^{-1}\left(f\left(w_{1}, w_{2}\right)-f\left(w_{1}, 0\right)\right) .
\end{aligned}
$$

Таким образом, все операторы деления являются непрерывными, откуда $\mathscr{O}_{\mathfrak{g}}\left(D_{r}\right)$ является слабо обращающим пополнением $\mathscr{U}(\mathfrak{g})$.

Теорема 3 доказана.

Теперь следует показать, что теорема 3 в свою очередь влечет, что каноническое вложение $\mathscr{U}(\mathfrak{g}) \rightarrow \mathscr{O}_{\mathfrak{g}}\left(D_{r}\right)$ - слабая локализация в смысле работы [15]; в частности, тривиальный $\mathscr{O}_{\mathfrak{g}}\left(D_{r}\right)$-модуль обладает резольвентой Кошуля, что мы продемонстрируем в следующем параграфе.

\section{§ 6. Резольвента Кошуля}

В этом параграфе мы докажем, что тривиальный модуль над $\mathscr{O}_{\mathfrak{g}}\left(D_{r}\right)$ обладает резольвентой Кошуля, что приведет к главному результату о трансверсальности. Сначала напомним некоторые основные определения топологической гомологии (см. [3])

6.1. Резольвента и трансверсальность. Пусть $A$ есть $\widehat{\otimes}$-алгебра. Проективное тензорное произведение (над $A$ ) модулей $X \in \bmod -A$ и $Y \in A$-mod обозначается $X \widehat{\otimes}_{A} Y$. По этому определению $X \widehat{\otimes}_{A} Y$ является пополнением факторпространства $X \widehat{\otimes} Y$ по замкнутому подпространству, порожденному элементами $x \cdot a \otimes y-x \otimes a \cdot y, x \in X, y \in Y, a \in A$. Говорят, что модуль $X \in A$-mod является свободным $A$-модулем, если $X=A \widehat{\otimes} E$ для некоторого полинормированного пространства $E$. Левая модульная структура на $A \widehat{\otimes} E$ задается правилом $a \cdot(b \otimes e)=a b \otimes e, a, b \in A, e \in E$. Говорят, что модуль $X \in A$-mod является проективным $A$-модулем, если он является ретрактом (в $A$-mod) некоторого свободного $A$-модуля. Проективная резольвента $A$-модуля $X$ - это комплекс $(\mathscr{P}, d)$ левых $A$-модулей с условием $\mathscr{P}_{n}=\{0\}$ для $n<0$ вместе с морфизмом $\epsilon: \mathscr{P}_{0} \rightarrow X$ такой, что комплекс

$$
0 \leftarrow X \stackrel{\epsilon}{\longleftarrow} \mathscr{P}_{0} \stackrel{d_{0}}{\longleftarrow} \mathscr{P}_{1} \stackrel{d_{1}}{\longleftarrow} \cdots
$$

допустим и все $\mathscr{P}_{n}$ являются проективными модулями. Если $B-\widehat{\otimes}$-алгебра и $F: A$-mod $\rightarrow B$-mod - аддитивный функтор, то через $F_{n}$ обозначим $n$-й (проективный) производный функтор от $F$. По определению (см. [3; п. 3.3.1]) $F_{n}(X)$ является как раз $n$-й гомологией цепного комплекса $(F(\mathscr{P}), F(d))$ для проективной резольвенты $(\mathscr{P}, d) A$-модуля $X$. Поскольку все проективные резольвенты модуля гомотопически эквивалентны (см. [3; п. 3.2.3]), легко видеть, 
что $F_{n}(X)$ не зависит от конкретного выбора проективной резольвенты $(\mathscr{P}, d)$ модуля $X$. Если $F$ является функтором $X \widehat{\otimes}_{A}$ о, то используем стандартное выражение $\operatorname{Tor}_{n}^{A}(X, \circ)$ для обозначения $n$-го производного функтора. Наконец, если $F: A$-mod $\rightarrow B$-mod является аддитивным функтором и $X \in A$-mod, то положим $F \perp X$, если $F_{n}(X)=\{0\}, n \geqslant 0$. Если $F=X \widehat{\otimes}_{A}$ 。, то пишем $X \perp Y$ (см. [1]) для $Y \in A$-mod в случае, когда $F \perp Y$. В этом случае говорят, что модули $X$ и $Y$ находятся в трансверсальном отношении. Отметим, что $X \perp Y$ тогда и только тогда, когда

$$
(\stackrel{\widehat{\otimes}}{A} Y) \perp X
$$

(см. [3; п. 3.4.26], [24]).

6.2. Резольвента для тривиального $\mathscr{O}_{\mathfrak{g}}\left(D_{r}\right)$-модуля. Пусть $A$ является пополнением $\mathscr{U}(\mathfrak{g})$. Таким образом, $A$ является алгеброй Фреше и существует плотный гомоморфизм алгебр $\iota: \mathscr{U}(\mathfrak{g}) \rightarrow A$ (см. п. 3.2). В частности, мы имеем левое $L_{\mathfrak{g}}: \mathfrak{g} \rightarrow \mathscr{L}(A), L_{\mathfrak{g}}(u) f=u \cdot f$, и правое $R_{\mathfrak{g}}: \mathfrak{g}^{\text {op }} \rightarrow \mathscr{L}(A), R_{\mathfrak{g}}(u) f=f \cdot u$, представления, т.е. $L_{\mathfrak{g}}(u)=L_{u}$ и $R_{\mathfrak{g}}(u)=R_{u}$. Комплексы $\operatorname{Kошуля~} \operatorname{Kos}\left(A, L_{\mathfrak{g}}\right)$ и $\operatorname{Kos}\left(A, R_{\mathfrak{g}}\right)$ являются соответственно правым и левым свободными комплексами $A$-модулей. Если $A=\mathscr{O}_{\mathfrak{g}}\left(D_{a, r}\right)$, то цепной комплекс $\operatorname{Kos}\left(\mathscr{O}_{\mathfrak{g}}\left(D_{a, r}\right), R_{\mathfrak{g}-a}\right)$ расширен посредством непрерывного характера $a: \mathscr{O}_{\mathfrak{g}}\left(D_{a, r}\right) \rightarrow \mathbb{C}(a)$, т.е.

$$
0 \leftarrow \mathbb{C}(a) \stackrel{a}{\longleftarrow} \operatorname{Kos}\left(\mathscr{O}_{\mathfrak{g}}\left(D_{a, r}\right), R_{\mathfrak{g}-a}\right)
$$

является комплексом в $\mathscr{O}_{\mathfrak{g}}\left(D_{a, r}\right)$-mod.

ПРЕДЛОЖЕНИЕ 2. Комплекс $\operatorname{Kos}\left(\mathscr{O}_{\mathfrak{g}}\left(D_{a, r}\right), L_{\mathfrak{g}-a}\right)$ (соответственно комплекс $\left.\operatorname{Kos}\left(\mathscr{O}_{\mathfrak{g}}\left(D_{a, r}\right), R_{\mathfrak{g}-a}\right)\right)$ является свободной резолъвентой правого (соответственно левого) тривиального $\mathscr{O}_{\mathfrak{g}}\left(D_{a, r}\right)$-модуля $\mathbb{C}(a)$.

ДоказАтельство. Можно считать, что $a=0$. По теореме $3 \mathscr{O}_{\mathfrak{g}}\left(D_{r}\right)$ - обращающее пополнение алгебры $\mathscr{U}(\mathfrak{g})$. Тогда $\operatorname{Kos}^{+}\left(\mathscr{O}_{\mathfrak{g}}\left(D_{r}\right), R_{\mathfrak{g}}\right)$ - допустимый цепной комплекс согласно теореме 2 . В частности, $\operatorname{im}\left(d_{0}\right)$ замкнут, где

$$
d_{0}: \mathscr{O}_{\mathfrak{g}}\left(D_{r}\right) \otimes \mathfrak{g} \rightarrow \mathscr{O}_{\mathfrak{g}}\left(D_{r}\right), \quad d_{0}(f \otimes u)=f u,
$$

является дифференциалом комплекса $\operatorname{Kos}\left(\mathscr{O}_{\mathfrak{g}}\left(D_{r}\right), R_{\mathfrak{g}}\right)$. Остается доказать, что

$$
\operatorname{im}\left(d_{0}\right)=\operatorname{ker}\left(\varepsilon_{\mathfrak{g}}\right),
$$

где $\varepsilon_{\mathfrak{g}}: \mathscr{O}_{\mathfrak{g}}\left(D_{r}\right) \rightarrow \mathbb{C}\left(\varepsilon_{\mathfrak{g}}\right)$ - тривиальный характер. Ясно, что $\operatorname{im}\left(d_{0}\right) \subseteq \operatorname{ker}\left(\varepsilon_{\mathfrak{g}}\right)$, и для каждого $f \in \mathscr{U}(\mathfrak{g})$ справедливо $f-\varepsilon_{\mathfrak{g}}(f) \cdot 1_{\mathscr{O}_{\mathfrak{g}}\left(D_{r}\right)} \in \operatorname{im}\left(d_{0}\right)$. Но последнее справедливо для всех $f \in \mathscr{O}_{\mathfrak{g}}\left(D_{r}\right)$, потому что $\operatorname{im}\left(d_{0}\right)$ замкнут и $\varepsilon_{\mathfrak{g}}$ непрерывен. Следовательно, $f \in \operatorname{im}\left(d_{0}\right)$, если $\varepsilon_{\mathfrak{g}}(f)=0$, т.е. $\operatorname{im}\left(d_{0}\right)=\operatorname{ker}\left(\varepsilon_{\mathfrak{g}}\right)$, откуда комплекс (7) допустим. Наконец, топологический изоморфизм $\widehat{\varkappa}_{\mathfrak{g}}: \mathscr{O}_{\mathfrak{g}}\left(D_{r}\right) \rightarrow$ $\mathscr{O}_{\mathfrak{g}}\left(D_{r}\right)^{\text {op }}$ (см. п. 5.1) осуществляет изоморфизм комплексов $\operatorname{Kos}\left(\mathscr{O}_{\mathfrak{g}}\left(D_{r}\right), L_{\mathfrak{g}}\right)$ и $\operatorname{Kos}\left(\mathscr{O}_{\mathfrak{g}}\left(D_{r}\right), R_{\mathfrak{g}}\right)$. Следовательно, то же самое верно для $\operatorname{Kos}\left(\mathscr{O}_{\mathfrak{g}}\left(D_{r}\right), L_{\mathfrak{g}}\right)$.

Предложение 2 доказано. 


\section{§ 7. Главный результат}

Теперь мы докажем, что трансверсальность порождает резольвентные точки относительно спектра Тейлора.

Пусть $\alpha: \mathfrak{g} \rightarrow \mathscr{L}(X)$ является представлением Ли в пространстве Фреше $X$. Очевидно, $\alpha-\lambda: \mathfrak{g} \rightarrow \mathscr{L}(X)-$ представление Ли для каждого $\lambda \in \Delta(\mathfrak{g})$. Напомним (см. [9], [11]), что спектр Тейлора $\sigma(\mathfrak{g}, X)$ g-модуля $X$ определяется как множество таких $\lambda \in \Delta(\mathfrak{g})$, что комплекс $\operatorname{Kошуля~} \operatorname{Kos}(X, \alpha-\lambda)$ не точен.

ТеОРема 4. Пусть $\mathfrak{g}$-алгебра Гейзенберга, $X$ - банахов $\mathfrak{g}$-модуль, и пусть $D_{a, r}-$ полидиск в $\mathbb{C}^{3}$ с иентром в точке $а$. Если $\mathscr{O}_{\mathfrak{g}}\left(D_{a, r}\right) \perp X$, то а $\notin \sigma(\mathfrak{g}, X)$,

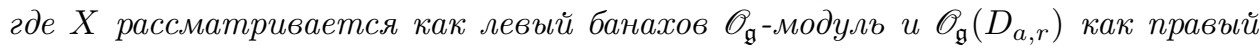
$\mathscr{O}_{\mathfrak{g}}$-модуль Фреше.

ДокАЗАТЕЛьство. Как изложено выше, можно считать, что $a=0$. Так как $\mathscr{L}(X)$ является банаховой алгеброй и $\mathscr{O}_{\mathfrak{g}}$ является оболочкой Аренса-Майкла алгебры $\mathscr{U}(\mathfrak{g})$ (см. п. 5.1), то из этого вытекает, что представление $\alpha: \mathfrak{g} \rightarrow \mathscr{L}(X)$, определенное посредством $\mathfrak{g}$-модуля $X$, может быть продолжено до непрерывного гомоморфизма алгебр $\mathscr{O}_{\mathfrak{g}} \rightarrow \mathscr{L}(X)$. Отсюда $X \in \mathscr{O}_{\mathfrak{g}}$-mod. Более того, имея в виду $\mathscr{O}_{\mathfrak{g}} \subseteq \mathscr{O}_{\mathfrak{g}}\left(D_{r}\right)$, мы заключаем, что $\mathscr{O}_{\mathfrak{g}}\left(D_{r}\right) \in \mathscr{O}_{\mathfrak{g}}$-mod- $\mathscr{O}_{\mathfrak{g}}$.

Теперь предположим, что $\mathscr{O}_{\mathfrak{g}}\left(D_{r}\right) \perp X$; это означает, что $\left(\right.$ о $\left.\widehat{\otimes}_{\mathscr{O}_{\mathfrak{g}}} X\right) \perp \mathscr{O}_{\mathfrak{g}}\left(D_{r}\right)$ (см. п. 6.1). По предложению 2 цепной комплекс

$$
0 \leftarrow \mathbb{C}\left(\varepsilon_{\mathfrak{g}}\right) \stackrel{\varepsilon_{\mathfrak{g}}}{\longleftarrow} \operatorname{Kos}\left(\mathscr{O}_{\mathfrak{g}}\left(D_{r}\right), L_{\mathfrak{g}}\right)
$$

правого $\mathscr{O}_{\mathfrak{g}}$-модуля является допустимым. Каждый член $\mathscr{O}_{\mathfrak{g}}\left(D_{r}\right) \otimes \wedge^{k} \mathfrak{g}$ комплекса $\operatorname{Kos}\left(\mathscr{O}_{\mathfrak{g}}\left(D_{r}\right), L_{\mathfrak{g}}\right)$ является топологической прямой суммой копий $\mathscr{O}_{\mathfrak{g}}\left(D_{r}\right)$. Поскольку ○ $\widehat{\otimes}_{\mathfrak{g}} X-$ аддитивный функтор, мы заключаем, что

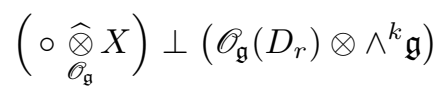

для всех $k$. Тогда $\left(\circ \widehat{\otimes}_{\mathscr{O}_{\mathfrak{g}}} X\right) \perp \mathbb{C}\left(\varepsilon_{\mathfrak{g}}\right)$ согласно [3; п. 3.3.8]. Следовательно, $\mathbb{C}\left(\varepsilon_{\mathfrak{g}}\right) \perp X$, где $\mathbb{C}\left(\varepsilon_{\mathfrak{g}}\right)$ рассматривается как правый $\mathscr{O}_{\mathfrak{g}}$-модуль. Таким образом, $\operatorname{Tor}_{n}^{\mathscr{O}_{\mathfrak{g}}}\left(\mathbb{C}\left(\varepsilon_{\mathfrak{g}}\right), X\right)=\{0\}$ для всех $k \geqslant 0$. Снова используя предложение 2 , но теперь для полидиска $D_{a, r}=\mathbb{C}^{3}$, мы получим, что комплекс

$$
0 \leftarrow \mathbb{C}\left(\varepsilon_{\mathfrak{g}}\right) \stackrel{\varepsilon_{\mathfrak{g}}}{\longleftarrow} \operatorname{Kos}\left(\mathscr{O}_{\mathfrak{g}}, L_{\mathfrak{g}}\right)
$$

правых $\mathscr{O}_{\mathfrak{g}}$-модулей допустим, т.е. $\operatorname{Kos}\left(\mathscr{O}_{\mathfrak{g}}, L_{\mathfrak{g}}\right)$ является свободной резольвентой правого $\mathscr{O}_{\mathfrak{g}}$-модуля $\mathbb{C}\left(\varepsilon_{\mathfrak{g}}\right)$. Отметим, что $\operatorname{Tor}_{n}^{\mathscr{O}_{\mathfrak{g}}}\left(\mathbb{C}\left(\varepsilon_{\mathfrak{g}}\right), X\right), k \geqslant 0$, являются гомологиями комплекса $\operatorname{Kos}\left(\mathscr{O}_{\mathfrak{g}}, L_{\mathfrak{g}}\right) \widehat{\otimes}_{\mathscr{O}_{\mathfrak{g}}} X$. Однако легко видеть, что $\operatorname{Kos}\left(\mathscr{O}_{\mathfrak{g}}, L_{\mathfrak{g}}\right) \widehat{\otimes}_{\mathscr{O}_{\mathfrak{g}}} X$ является в точности комплексом Кошуля $\operatorname{Kos}(X, \alpha) \mathfrak{g}$-модуля $X$. Отсюда получаем, что комплекс $\operatorname{Kos}(X, \alpha)$ точен, а это означает, что $0 \notin \sigma(\mathfrak{g}, X)$.

Теорема 4 доказана.

ЗАмЕчАниЕ 1. Этот же результат для нильпотентной алгебры Ли $\mathfrak{g}$ не доказан. В общем случае радикал Джекобсона $\operatorname{Rad} \mathscr{O}_{\mathfrak{g}}\left(D_{a, r}\right)$ имеет более сложную структуру, не такую, как в предложении 1. 
ЗАмЕчАНИЕ 2. Полное расщепление радикала $\operatorname{Rad} \mathscr{O}_{\mathfrak{g}}\left(D_{a, r}\right)$ в том смысле, что каждый $f \in \operatorname{Rad} \mathscr{O}_{\mathfrak{g}}\left(D_{a, r}\right)$ имеет безусловное разложение $f=\sum_{k \geqslant 1} f_{k} z^{k}$, $f_{k} \in \mathscr{S}_{e}\left(D_{a, r}\right)$, повлекло бы обратный теореме 4 результат, а именно: если $a \notin \sigma(\mathfrak{g}, X)$, то $\mathscr{O}_{\mathfrak{g}}\left(D_{a, r}\right) \perp X$ для достаточно малого полидиска $D_{a, r}$ с центром в точке $a$. Все эти утверждения и многие другие могут быть получены в формально-радикальных пополнениях $\mathscr{U}(\mathfrak{g})$ (см. [18], [19]), но они существенно ограничивают класс рассматриваемых банаховых $\mathfrak{g}$-модулей $X$, т.е. операторные пары $(T, S)$, порождающие алгебры Гейзенберга. Точнее, приходится иметь дело с операторной парой $(T, S)$ такой, что $[T,[T, S]]=[S,[T, S]]=0$ и $[T, S]$ нильпотентен. Это частный случай так называемой супернильпотентной операторной алгебры Ли, для которой некоммутативное голоморфное функциональное исчисление, расширяющее исчисление Тейлора (см. [1]), имеет место (см. [21]).

\section{Список литературы}

[1] J. L. Taylor, "A general framework for a multi-operator functional calculus", Advances in Math., 9:2 (1972), 183-252.

[2] J. L. Taylor, "Homology and cohomology for topological algebras", Advances in Math., 9:2 (1972), 137-182.

[3] А.Я. Хелемский, Гомология в банаховых и топологических алгебрах, Изд-во Моск. ун-та, М., 1986; англ. пер.: А. Ya. Khelemskii, The homology of Banach and topological algebras, Math. Appl. (Soviet Ser.), 41, Kluwer Acad. Publ., Dordrecht, 1989.

[4] В. П. Маслов, Операторные методы, Наука, М., 1973; англ. пер.: V. P. Maslov, Operational methods, Mir, Moscow, 1976.

[5] J. L. Taylor, "A joint spectrum for several commuting operators", J. Functional Analysis, 6:2 (1970), 172-191.

[6] J. L. Taylor, "The analytic-functional calculus for several commuting operators", Acta Math., 125:1 (1970), 1-38.

[7] J. Eschmeier, M. Putinar, Spectral decompositions and analytic sheaves, London Math. Soc. Monogr. (N.S.), 10, Clarendon Press, Oxford Univ. Press, Oxford, 1996.

[8] E. Boasso, "Dual properties and joint spectra for solvable Lie algebras of operators", J. Operator Theory, 33:1 (1995), 105-116.

[9] A. S. Fainshtein, "Taylor joint spectrum for families of operators generating nilpotent Lie algebras", J. Operator Theory, 29:1 (1993), 3-27.

[10] A. Dosiev, "Spectra of infinite parametrized Banach complexes", J. Operator Theory, 48:3 (2002), 585-614.

[11] A. Dosiev, "Cartan-Slodkowski spectra, splitting elements and noncommutative spectral mapping theorems", J. Funct. Anal., 230:2 (2006), 446-493.

[12] A. Dosiev, "Quasispectra of solvable Lie algebra homomorphisms into Banach algebras", Studia Math., 174:1 (2006), 13-27.

[13] А.А. Досиев, "Голоморфные функции от базиса нильпотентной алгебры Ли", Функи. анализ и его прил., 34:4 (2000), 82-84; англ. пер.: А. А. Dosiev, "Holomorphic functions of a basis of a nilpotent Lie algebra", Funct. Anal. Appl., 34:4 (2000), 302-304.

[14] А.А. Досиев, "Алгебры степенных рядов от элементов алгебры Ли и спектры Слодковского", Исследования по линейным операторам и теории функиий. 30, Зап. научн. сем. ПОМИ, 290, ПОМИ, СПб., 2002, 72-121; англ. пер.: A. Dosiev, 
"Algebras of power series of elements of a Lie algebra and the Slodkowski spectra", J. Math. Sci. (N. Y.), 124:2 (2004), 4886-4908.

[15] A. Yu. Pirkovskii, "Stably flat completions of universal enveloping algebras", Dissertationes Math. (Rozprawy Mat.), 441 (2006); arXiv: math/0311492v2.

[16] А.Я. Хелемский, Банаховы и полинормированные алгебры: общая теория, представления, гомология, Наука, М., 1989.

[17] А. А. Доси, "Некоммутативные голоморфные функции от элементов алгебры Ли и задача об абсолютном базисе", Изв. РАН. Сер. матем., 73:6 (2009), 77-100; англ. пер.: А. A. Dosi, "Non-commutative holomorphic functions in elements of a Lie algebra and the absolute basis problem", Izv. Math., 73:6 (2009), 1149-1171.

[18] A. A. Dosiev, "Formally-radical functions in elements of a nilpotent Lie algebra and noncommutative localizations", Algebra Colloquium (to appear).

[19] A. Dosi, "Fréchet sheaves and Taylor spectrum for supernilpotent Lie algebras of operators", Mediterr. J. Math., 6:2 (2009), 181-201.

[20] M. Kapranov, "Noncommutative geometry based on conductor expansions", J. Reine Angew. Math., 505 (1998), 73-118.

[21] А.А. Досиев, "Когомологии пучков алгебр Фреше и спектральная теория", Функи. анализ и его прил., 39:3 (2005), 76-80; англ. пер.: А.А. Dosiev, "Cohomology of sheaves of Frechet algebras and spectral theory", Funct. Anal. Appl., 39:3 (2005), 225-228.

[22] A. A. Dosi, "Taylor functional calculus for supernilpotent Lie algebra of operators", J. Operator Theory, 2010 (to appear).

[23] А. А. Досиев, "Гомологические размерности алгебры целых функций от элементов нильпотентной алгебры Ли", Функи. анализ и его прил., 37:1 (2003), 73-77; англ. пер.: А. A. Dosiev, "Homological dimensions of the algebra formed by entire functions of elements of a nilpotent Lie algebra", Funct. Anal. Appl., 37:1 (2003), 61-64.

[24] A. Dosiev, "Local left invertibility for operator tuples and noncommutative localizations", J. K-Theory, 4:1 (2009), 163-191.

[25] Ж. Диксмье, Универсальные обертывающие алгебры, Мир, М., 1978; пер. с фр.: J. Dixmier, Algèbres enveloppantes, Gauthier-Villars, Paris-Brussels-Montreal, 1974.

А. А. Доси (А. A. Dosi)

Middle East Technical University

Northern Cyprus Campus, Güzelyurt, Turkey

E-mail: dosiev@yahoo.com
Поступила в редакцию

02.10 .2008 и 14.10 .2009 